\title{
Computation of Dynamic Derivatives Using CFD
}

\author{
A. Da Ronch, ${ }^{*}$ D. Vallespin, ${ }^{\dagger}$ M. Ghoreyshi, ${ }^{\ddagger}$ and K.J. Badcock, ${ }^{\S}$ \\ Department of Engineering, University of Liverpool, Liverpool, UK L69 3BX, United Kingdom
}

\begin{abstract}
This paper focuses on the computation of dynamic derivatives for full aircraft configurations. The flow is modelled using the Euler and RANS equations and an unsteady time-domain solver is used for the computation of aerodynamic loads for forced periodic motions. The study investigates the variation of damping values through the transonic regime and for several permutations of motion parameters for the Standard Dynamics Model geometry. A benchmark against experimental data is presented for the Transonic CRuiser wind tunnel geometry. For the SDM, strake vortices and their breakdown are observed when increasing the mean angle of attack during the applied pitch sinusoidal motion. A good agreement is obtained with available experimental data. For the TCR, a validation of longitudinal aerodynamic characteristics is first considered. Numerical experiments for the estimation of damping derivatives and for large amplitude forced oscillations in pitch axis are compared to wind tunnel data. Simulations are in agreement with experimental data up to high angles of attack.
\end{abstract}

\section{Introduction}

The increasing manoeuvre capabilities of modern combat aircraft have highlighted the limitations and shortcomings of the conventional stability or aerodynamic derivatives based model for the representation of the aerodynamic loads in the aircraft equations of motion. An important consideration is the presence of significant motion frequency effects on the dynamic derivatives measured in small-amplitude oscillatory wind tunnel tests at higher angles of attack. This frequency dependence cannot be reconciled with the stability derivatives model. Although these effects were first recognised in the 1950s, they played in general an insignificant role for conventional aircraft operating at benign conditions. The changing interest in the determination of dynamic stability derivatives due to the requirements of increasing angle of attack and Mach numbers during the 1970s is described in Orlik-Rückemann. ${ }^{1}$ Modern combat aircraft are capable of performing agile manoeuvres involving high pitch rates at extreme angles of attack. Vehicles manoeuvring in this regime are subject to non-linear aerodynamic loads. The non-linearities are mainly due to three dimensional separated flow and concentrated vortices that are generated. The appearance of these flows alters the dynamic behaviour, in ways that are not predictable on the basis of linearised formulations of the aerodynamic forces and moments. Accurate prediction of the non-linear airloads is of great importance in the analysis of aircraft flight motion and in the design of suitable flight control systems.

The concept of stability or aerodynamic derivatives was introduced by Bryan ${ }^{2}$ in 1911 and remains essentially unchanged as the conventional model for the representation of the aerodynamic loads in the equations of motion. It is assumed that the aerodynamic forces and moments are a function of the instantaneous values of the disturbance velocities, control angles and their rates. The dependence of the forces and moments on these variables is obtained by a Taylor series expansion, discarding higher order terms. ${ }^{3}$ For slow motions at low angle of attack, the static derivatives are generally sufficient to model the aerodynamic loads. At higher angles of attack and rates, Nguyen ${ }^{4}$ reported that the inclusion of dynamic derivatives in the aerodynamic model can have a significant effect on the calculated stability characteristics of an airframe. For an extended period of time, the aerodynamic functions were approximated by use of linear expressions leading to a concept of stability and control derivatives. The addition of non-linear terms to take into account

\footnotetext{
*Ph.D. Student, corresponding author. Tel.: +44(0)151-7948035, Email: A.Da-Ronch@liverpool.ac.uk

$\dagger$ Ph.D. Student

${ }^{\ddagger}$ Research Assistant

$\S$ Professor, SMAIAA
} 
changes of stability derivatives with the angle of attack extended the range of flight conditions to high angles of attack and/or high amplitude manoeuvres. In the linear and non-linear methods, it is assumed that the aerodynamic parameters are time invariant. This assumption was often questioned based on many studies of unsteady aerodynamics. In the 1920s, Wagner ${ }^{5}$ conducted a series of studies for the unsteady lift generated on an airfoil due to abrupt changes in angle of attack. Theodorsen extended these studies investigating the forces and moments on an oscillating airfoil. The lift response of an airfoil penetrating a sharp-edge gust and harmonically-varying gust was studied by Küssner ${ }^{6}$ and Sears, respectively. The first attempts to investigate unsteady aerodynamic effects on aircraft motion were made by Jones, ${ }^{7}$ studying the effect of the wing wake on the lift of the horizontal tail. A more general formulation of linear unsteady aerodynamics in the aircraft longitudinal equations of motion was introduced by Tobak. ${ }^{8}$ Tobak and Schiff ${ }^{9}$ extended the concept of linear aerodynamic formulation into the non-linear regime by replacing the indicial functions within the integrals with functionals, ${ }^{10}$ themselves dependent of the past motion. A different approach was proposed by Goman et al. ${ }^{11}$ introducing additional state variables, named internal state variables, in the functional relationships for the aerodynamic forces and moments. Coordinates of separation point or vortex breakdown can be taken as internal state variables. These are modelled by differential equations. Goman and Khrabrov ${ }^{12}$ formulated state space models with internal state variables describing the flow state. A good agreement was achieved with experimental data for a separated flow about an airfoil and flow with vortex breakdown about a slender delta wing.

Wind tunnel testing has traditionally been used to produce derivatives for production aircraft based on scale models. This can be a time consuming and expensive process. The physical realism of wind tunnel data is well known, but can be limited by blockage, scaling, and Reynolds-number effects together with support interference issues that prevent the proper modelling of the full-scale vehicle behaviour. Navier-Stokes CFD solvers have reached a level of robustness and maturity to support routine use on relatively inexpensive computer clusters. The computation of static stability derivatives can be done with present off-the-shelf CFD tools. The prediction of dynamic derivatives requires the ability to compute the aerodynamic response to time-dependent prescribed motions which are used to excite the aerodynamics of interest. CFD has potential for complementing experimental testing techniques for obtaining these aerodynamic parameters. The physical limitations and kinematic restrictions of wind tunnel testing including model motion as well as the interference effects of the model support are not factors in the computational analysis. Physical effects can be separated from the CFD solutions in a way which can be difficult from wind tunnel or flight test data. CFD can also be used for investigating the modelling of data from flight tests.

The current paper investigates the use of Computational Fluid Dynamics (CFD) in the prediction of dynamic derivatives for full aircraft configurations. The influence of applied sinusoidal motion parameters and flight conditions on the damping values is also addressed. The paper continues with the formulation of the underlying CFD solver. A review of the dynamic derivatives formulation is given and techniques to accurately compute these terms are defined. Results are presented for the Standard Dynamic Model (SDM) and the Transonic CRuiser (TCR).

\section{CFD Solver Formulation}

The flow solver used in the present study is the University of Liverpool PMB (Parallel Multi-Block) solver. The Euler and RANS equations are discretised on curvilinear multi-block body conforming grids using a cell-centred finite volume method which converts the partial differential equations (PDE's) into a set of ordinary differential equations (ODE's). The equations are solved on block structured grids using an implicit solver. A wide variety of unsteady flow problems, including aeroelasticity, cavity flows, aerospike flows, delta wing aerodynamics, rotorcraft problems and transonic buffet have been studied by means of this code. A validation against flight data for the F-16XL aircraft has also been performed. ${ }^{13}$ The main features of the CFD solver are detailed in Badcock et al. ${ }^{14}$ The applications presented in the current paper model the flow using the Euler equations for the SDM and the RANS equations for the TCR.

The three-dimensional Navier-Stokes equations may be written in non-dimensional conservative form as

$$
\frac{\partial \mathbf{W}}{\partial t}+\frac{\partial\left(\mathbf{F}^{i}+\mathbf{F}^{v}\right)}{\partial x}+\frac{\partial\left(\mathbf{G}^{i}+\mathbf{G}^{v}\right)}{\partial y}+\frac{\partial\left(\mathbf{H}^{i}+\mathbf{H}^{v}\right)}{\partial z}=0
$$

where $\mathbf{W}$ is the vector of conserved variables, $\mathbf{W}=[\rho, \rho u, \rho v, \rho w, \rho E]^{T}, \rho$ is the density and $u, v$, and $w$ are the components of velocity given by the Cartesian velocity vector $\mathbf{U}=(u, v, w)^{T}$. The total energy per 
unit mass is $E$. The superscripts $\left({ }^{i}\right)$ and $\left({ }^{v}\right)$ denote the inviscid and viscous components of the flux vectors, $\mathbf{F}$ ( $x$-direction), $\mathbf{G}$ ( $y$-direction) and $\mathbf{H}$ ( $z$-direction). The inviscid flux vectors, $\mathbf{F}^{i}, \mathbf{G}^{i}$ and $\mathbf{H}^{i}$, are given by

$$
\begin{aligned}
& \mathbf{F}^{i}=\left(\rho u, \rho u^{2}+p, \rho u v, \rho u w, u(\rho E+p)\right)^{T} \\
& \mathbf{G}^{i}=\left(\rho v, \rho v u, \rho v^{2}+p, \rho v w, v(\rho E+p)\right)^{T} \\
& \mathbf{H}^{i}=\left(\rho w, \rho w u, \rho w v, \rho w^{2}+p, w(\rho E+p)\right)^{T}
\end{aligned}
$$

while the viscous flux vectors, $\mathbf{F}^{v}, \mathbf{G}^{v}$ and $\mathbf{H}^{v}$, contain terms of the heat flux and viscous forces exerted on the body and can be represented by

$$
\begin{aligned}
\mathbf{F}^{v} & =\frac{1}{R e}\left(0, \tau_{x x}, \tau_{x y}, \tau_{x z}, u \tau_{x x}+v \tau_{x y}+w \tau_{x z}+q_{x}\right)^{T} \\
\mathbf{G}^{v} & =\frac{1}{R e}\left(0, \tau_{x y}, \tau_{y y}, \tau_{y z}, u \tau_{x y}+v \tau_{y y}+w \tau_{y z}+q_{y}\right)^{T} \\
\mathbf{H}^{v} & =\frac{1}{R e}\left(0, \tau_{x z}, \tau_{y z}, \tau_{z z}, u \tau_{x z}+v \tau_{y z}+w \tau_{z z}+q_{z}\right)^{T}
\end{aligned}
$$

The stress tensor components, $\tau_{i j}$, and the heat flux vector components, $q_{i}$, can be found in numerous text books such as Anderson. ${ }^{15}$ The Navier-Stokes equations are discretised using a cell-centred finite volume approach. The computational domain is divided into a finite number of non-overlapping control volumes and the governing equations are applied to each cell in turn. A fully implicit steady solution of the RANS equations is obtained by advancing the solution forward in time by solving the discrete non-linear system of equations

$$
\frac{\mathbf{W}_{i j k}^{n+1}-\mathbf{W}_{i j k}^{n}}{\Delta t}=\frac{1}{V_{i j k}} \mathbf{R}\left(\mathbf{W}_{i j k}^{n+1}\right)
$$

where $V_{i j k}$ denotes the cell volume, $\mathbf{W}_{i j k}$ represents the flux variables and $\mathbf{R}\left(\mathbf{W}_{i j k}^{n+1}\right)$ the flux residuals. The pseudo time step is indicated by $\Delta t$. Eq. (4) represents a system of non-linear algebraic equations and to simplify the solution procedure, the flux residual is linearised in time. The flux residual is the discretisation of the convective terms, given here by Osher's approximate Riemman solver, ${ }^{16}$ MUSCL interpolation, ${ }^{17}$ and Van Albada's limiter. An iterative Generalised Conjugate Gradient method is used to solve the linear system as it is capable of solving sparse equations efficiently in terms of time and memory requirements. A Block Incomplete Lower-Upper (BILU) factorisation method is used as pre-conditioner for the system of equations.

The implicit dual-time method proposed by Jameson ${ }^{18}$ is used for time-accurate calculations. The solution marches in pseudo-time for each real time-step to achieve convergence. In the current application, a key functionality of the CFD solver is the ability to move the mesh conforming the motion of the body. Rigid body motions can be treated by moving the mesh rigidly in response to the applied sinusoidal motion. The mesh is deformed once per real time step during the unsteady calculation. A curvilinear time dependent formulation is used to formulate the mapping between the computational space, uniform and Cartesian, and the physical space.

Due to the fact that the formulation of most turbulence models can also be represented in vector form, similar to Eq. (1), the steady state solver for the turbulence model equations is formulated and solved in a similar manner to the mean flow as described, with the vector $\mathbf{W}$ replaced by the equivalent turbulent vector and an equivalent substitution for the flux residual. For the turbulence model equations the flux residual also contains the dissipation source term, however the production term is treated explicitly. The eddy viscosity is calculated from the turbulent quantities as specified by the model and is used to advance the mean flow solution. This new mean flow solution is then used to update the turbulence solution, freezing the mean flow values. Several one- and two-equation turbulence models are available in the PMB solver. Among others, the one-equation Spalart-Allmaras model, ${ }^{19}$ the two-equation $k-\omega$ model proposed by Wilcox ${ }^{20}$ and the $k-\omega$ with $P_{\omega}$ enhancer proposed by Brandsma et al. ${ }^{21}$

\section{Calculation of Dynamic Derivatives}

\section{A. Oscillatory Data}

The estimation of the quasi-steady dynamic derivatives is obtained by imposing a forced sinusoidal motion around the aircraft centre of gravity. For the computation of the longitudinal dynamic derivative values 
from the time-histories of the forces and moments, it is assumed that the aerodynamic coefficients are linear functions of the angle of attack, $\alpha$, pitching angular velocity, $q$, and rates, $\dot{\alpha}$ and $\dot{q}$. To illustrate, the increment in the longitudinal aerodynamic coefficients (lift, drag and pitching moment) with respect to its mean value during the applied pitching sinusoidal motion is formulated as

$$
\Delta C_{j}=C_{j_{\alpha}} \Delta \alpha+\frac{l}{V} C_{j_{\dot{\alpha}}} \dot{\alpha}+\frac{l}{V} C_{j_{q}} q+\left(\frac{l}{V}\right)^{2} C_{j_{\dot{q}}} \dot{q}
$$

where $V$ is the magnitude of the aircraft velocity vector and $l$ is a characteristic length, defined as one half of the wing mean aerodynamic chord, $l=\bar{c} / 2$. The lift, drag and pitching moment coefficients are indicated setting the subscript $j=L, D, m$, respectively. The harmonic motion in pitch defines the kinematic relations for the angle of attack, pitching angular velocity and rate as

$$
\begin{aligned}
& \Delta \alpha=\alpha_{A} \sin (\omega t) \\
& \dot{\alpha}=q=\omega \alpha_{A} \cos (\omega t) \\
& \ddot{\alpha}=\dot{q}=-\omega^{2} \alpha_{A} \sin (\omega t)
\end{aligned}
$$

Eq. (5) can then be rewritten as

$$
\Delta C_{j}=\alpha_{A}\left(C_{j_{\alpha}}-k^{2} C_{j_{\dot{q}}}\right) \sin (\omega t)+\alpha_{A} k\left(C_{j_{\dot{\alpha}}}+C_{j_{q}}\right) \cos (\omega t)
$$

where $k=l \omega / V$ indicates the non-dimensional reduced frequency of the applied motion. The in-phase and out-of-phase components of $\Delta C_{j}$, respectively indicated as $\bar{C}_{j_{\alpha}}$ and $\bar{C}_{j_{q}},{ }^{22}$ are defined as

$$
\begin{aligned}
\bar{C}_{j_{\alpha}} & =\left(C_{j_{\alpha}}-k^{2} C_{j_{\dot{q}}}\right) \\
\bar{C}_{j_{q}} & =\left(C_{j_{\dot{\alpha}}}+C_{j_{q}}\right)
\end{aligned}
$$

and then

$$
\Delta C_{j}=\alpha_{A} \bar{C}_{j_{\alpha}} \sin (\omega t)+\alpha_{A} k \bar{C}_{j_{q}} \cos (\omega t)
$$

The out-of-phase component is sometimes referred to as the in-quadrature component. ${ }^{3}$ The dynamic derivative values can be calculated taking the first Fourier coefficients of the time history of $\Delta C_{j}$ over $n_{c}$ cycles

$$
\begin{aligned}
\bar{C}_{j_{\alpha}} & =\frac{2}{\alpha_{A} n_{c} T} \int_{0}^{n_{c} T} \Delta C_{j}(t) \sin (\omega t) d t \\
\bar{C}_{j_{q}} & =\frac{2}{k \alpha_{A} n_{c} T} \int_{0}^{n_{c} T} \Delta C_{j}(t) \cos (\omega t) d t
\end{aligned}
$$

where $T=2 \pi / \omega$ is the period of one cycle of unsteadiness. If the aerodynamic model formulated in Eq. (5) is correct, the plots $\Delta C_{j}$ versus $\alpha(t)$ should resemble ellipses. In applications of common interest the aerodynamic loads are not linear functions of the instantaneous flight states. The flow solution of the NACA 0012 airfoil for the transonic AGARD CT 5 case reported in Da Ronch et al. ${ }^{23}$ is non-linear, with a shock appearing in the leading edge region and moving downstream until approximately $45 \%$ of the chord. At moderate angle of incidence, the development and breakdown of vortices is influenced by the airframe motion as shown below for the SDM and TCR configurations.

The in-phase component is comprised of a static derivative and a rotational derivative, whereas the outof-phase component includes a rotary derivative and a translation acceleration derivative. The frequency effect is accounted for explicitly in the equations for the in-phase component, while the equations for the out-of-phase component used to determine the damping derivatives do not include the frequency effect. The frequency effect on dynamic derivatives is generally insignificant for conventional aircraft operating at benign flight conditions. However, fighter configurations are designed to operate at extreme angles of incidence performing high rate manoeuvres in flight regions where the frequency effects are evident. ${ }^{22,24}$ Models for an aircraft performing a one degree of freedom oscillatory motion in either roll and yaw can be developed in a similar fashion to that for the pitching oscillations. ${ }^{25,26}$ 


\section{Fourier Integral}

In the current paper, the computation of the in-phase and out-of-phase components of the aerodynamic coefficients, as formulated in the integrals in Eq. (11) and (12), is performed by a numerical technique ${ }^{27}$ applied to the last cycles of the steady harmonic outputs. Frequency domain analysis has many advantages and is currently used in different research areas. ${ }^{28}$ The transformation of the sampled time domain data into the frequency domain is achieved by an approximation of the finite Fourier integral. The finite Fourier integral of a continuous scalar time function $x(t)$ on a finite time interval, $t \in[0, T]$, is defined as

$$
X(f)=\int_{0}^{T} x(t) e^{-i 2 \pi f t} d t
$$

where $i$ is the imaginary unit and $f$ the frequency in $[\mathrm{Hz}]$. Accuracy of the transformation to the frequency domain can be improved using quadrature methods. Filon ${ }^{29}$ proposed in the 1920 s an interpolation scheme to improve the accuracy of the finite Fourier integral. The finite Fourier integral is evaluated at discrete values of frequency, $f_{k}$, evenly spaced between zero frequency and the Nyquist frequency, $f_{N}$, with a frequency resolution equal to the reciprocal of the length of the time record. ${ }^{30}$ Arbitrary frequency resolution in a selected frequency band of interest can be obtained using the Chirp z-transform, ${ }^{31}$ decoupling the frequency resolution from the length of the time record. A cubic Lagrange polynomial interpolation scheme is implemented in the present framework to achieve accurate evaluations of the in-phase and out-of-phase components. A Chirp z-cubic approximation to the finite Fourier integral is also available.

\section{Least Squares Solution}

An alternative approach to the one outlined above leads to the solution of a least squares problem. ${ }^{32}$ Eq. (5) can be formulated within a general framework as

$$
y=a_{0}+a_{1} x_{1}+a_{2} x_{2}+\ldots+a_{p} x_{p}+e
$$

for a dependent variable $y$ and the independent arguments, $x_{1}, x_{2}, \ldots, x_{p}$, where $p$ is the number of independent variables. Here $a_{0}, a_{1}, \ldots, a_{p}$ are unknown parameters of the mathematical model and $e$ is the approximation error. The dependent variable represents the dependency upon time of the integrated aerodynamic coefficients. In the contest of small amplitude pitch oscillations, the independent arguments are the instantaneous angle of attack and its rate of change. The parameters of the mathematical model are estimated using the $n$ values of the instantaneous numerical measurements of the $p+1$ variables, $y, x_{1}, x_{2}, \ldots, x_{p}$. The vector of the dependent variable sampled in time and the vector of unknown regression parameters are denoted, respectively, by $\mathbf{y}=\left(y_{1}, y_{2}, \ldots y_{n}\right)^{T}$ and $\mathbf{x}=\left(x_{1}, x_{2}, \ldots, x_{p}\right)^{T}$. The vector of approximation errors is $\mathbf{e}=\left(e_{1}, e_{2}, \ldots, e_{p}\right)^{T}$ and the matrix relating the unknowns with the independent variable, with dimensions $(n, p+1)$, is given by

$$
\mathbf{A}=\left[\begin{array}{cccc}
1 & a_{11} & \ldots & a_{p 1} \\
1 & a_{12} & \ldots & a_{p 2} \\
\vdots & \vdots & \ddots & \vdots \\
1 & a_{1 n} & \ldots & a_{p n}
\end{array}\right]
$$

The corresponding linear regression model is given by Eq. (16).

$$
\mathbf{y}=\mathbf{A} \mathbf{x}+\mathbf{e}
$$

The unknown vector of the approximate solution which minimizes the sum of the squares of the $e_{i}$ is found by minimizing the functional $J=1 / 2\|e\|^{2}$

$$
\frac{\partial J}{\partial x_{i}}=0, i=0,1, \ldots, p
$$

which results in $\left(\mathbf{A}^{T} \mathbf{A}\right) \mathbf{x}=\mathbf{A}^{T} \mathbf{y}$. The use of the linear regression technique provides the estimation of the aerodynamic derivatives in small amplitude pitch oscillations.

Unsteady numerical data were processed for the estimation of dynamic derivatives using the two procedures. It was observed that nearly identical results were obtained in all test cases. 


\section{Test Cases}

\section{A. Standard Dynamics Model Aircraft}

The Standard Dynamics Model (SDM) is a generic fighter configuration based loosely on the F-16 planform. The model includes a slender strake-delta wing with leading-edge extensions (LEX), horizontal and vertical stabilizers, ventral fins and a blocked off inlet (Fig. 1). Further details on the geometry can be found in Huang. ${ }^{33}$ The SDM configuration has been tested extensively at various wind tunnel facilities to compare different measurement techniques. ${ }^{34-37}$

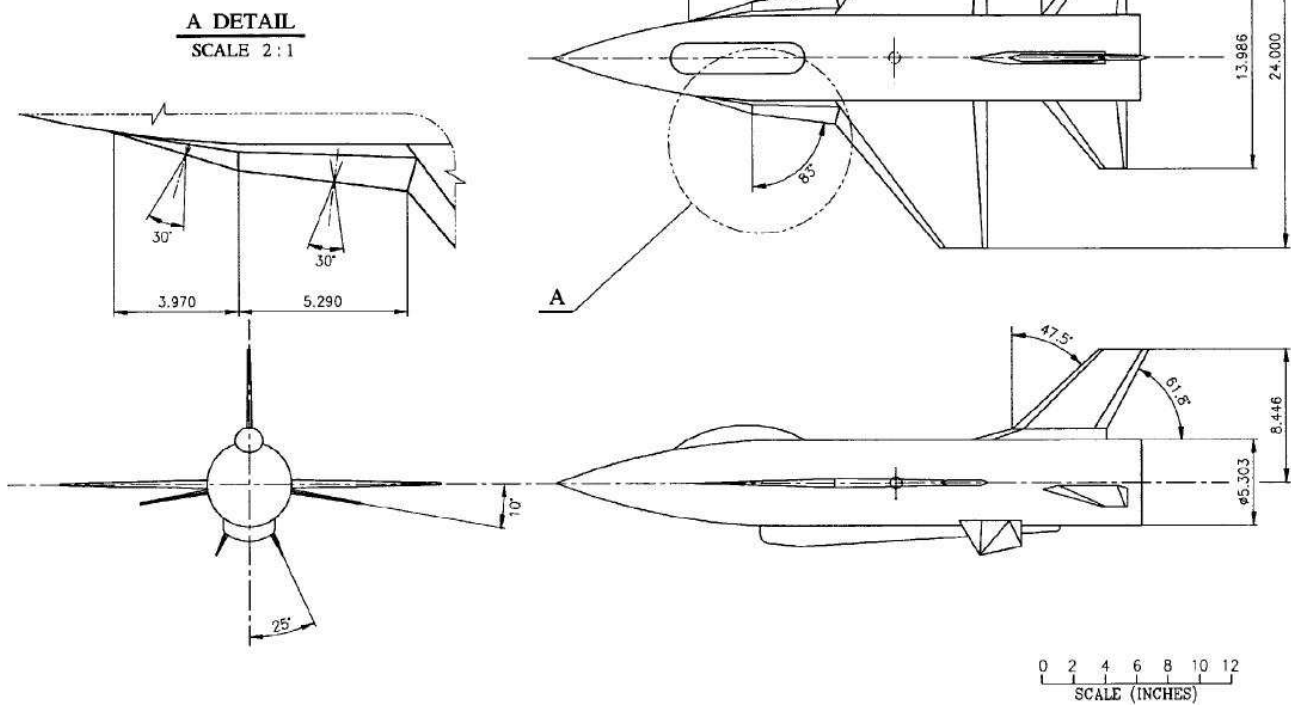

Figure 1. Standard Dynamic Model (SDM) layout from Huang ${ }^{33}$

A block structured mesh was generated, shown in Fig. 2. The geometry was slightly simplified by removing the blocked off intake and the ventral fins. These were considered reasonable simplifications because the main interest here is on the impact of the vortical flow developments on the upper lifting surfaces. A fine Euler mesh was generated with 2.4 million points representing one half of the SDM configuration. A coarse mesh for the full configuration was obtained with 701 thousand points by omitting every second point in each direction. All lifting surfaces have sharp-leading edges, which is beneficial for the CFD calculations.

The SDM model geometry has the dimensions specified in Table 2. The reference point for the moments is located at the centre of motion, which is positioned on the fuselage centreline at $55 \%$ along the fuselage length from the foremost point. Results for the coarse and half-fine grids are presented and indicated, respectively, by $C$ and $H F$.

\begin{tabular}{ll}
\hline \hline Reference wing area, $S$ & $0.1238 \mathrm{~m}^{2}$ \\
Reference wing span, $b$ & $0.6096 \mathrm{~m}$ \\
Mean aerodynamic chord, $\bar{c}$ & $0.2299 \mathrm{~m}$ \\
Fuselage total length & $0.9429 \mathrm{~m}$ \\
\hline \hline
\end{tabular}

Table 1. Reference values of the SDM model geometry 


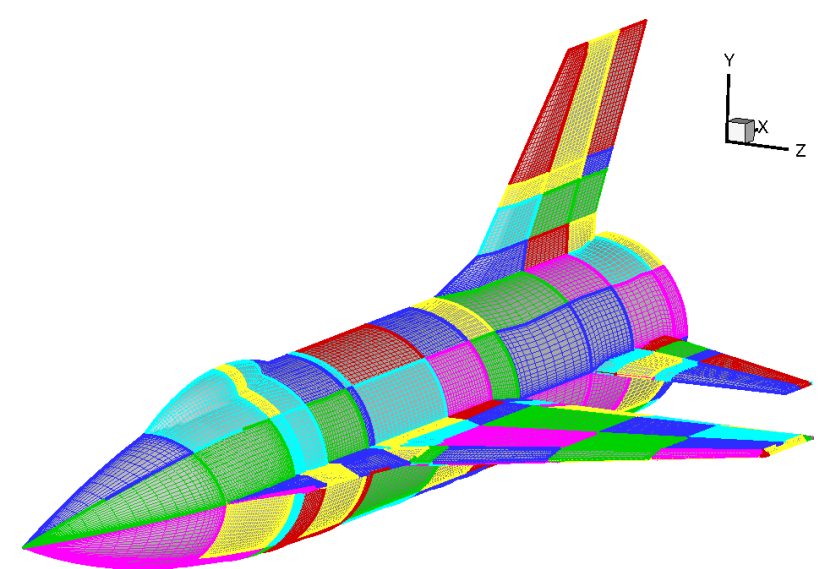

(a) Half fine, 2.4 million points

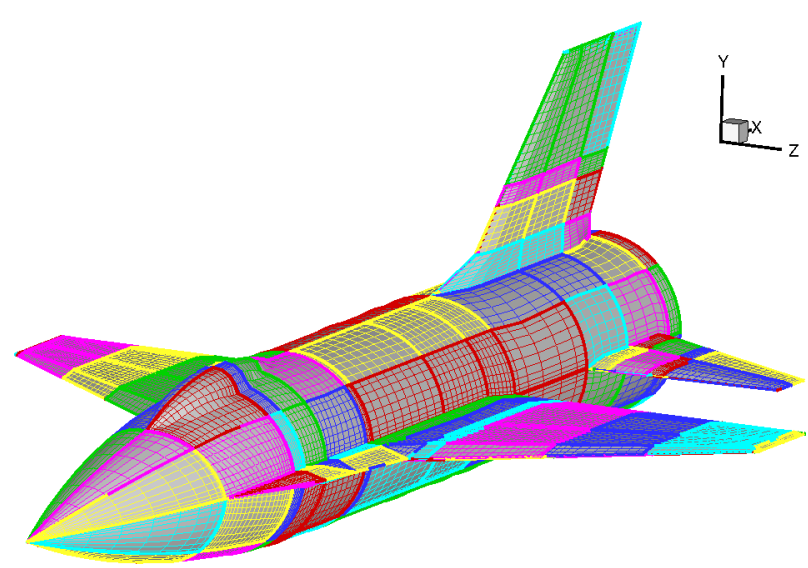

(b) Coarse, 0.7 million points

Figure 2. Surface grid for the SDM model geometry

\section{B. Transonic CRuiser Aircraft}

The design of the Transonic CRuiser aircraft was developed within the SimSAC project. ${ }^{38}$ The TCR model was computed using PMB on a structured multiblock grid generated using ICEM ANSYS. ${ }^{39}$ The model consists of an 8.5 million point mesh for the half configuration arranged in 675 blocks. The main characteristics of the mesh are illustrated in Fig. 3. The full mesh around the solid model and the symmetry plane are shown in Fig. 3(b), where the high concentration of cells in the regions adjacent to solid can be seen. The non-dimensional minimum spacing normal to the solid wall is of $2.5 \times 10^{-6}$ which allows for flows with Reynolds numbers of around 2 million to be simulated. An example of this cell concentration near the solid wall is included where the mesh on a section on the main wing is shown. The tips of the wing and the canard of the TCR model are both blunt, for which the same block topology was chosen. Here, a diamond shaped block fits into the leading edge part and another on the trailing one. The quality of the cells in these two areas is slightly compromised in order to allow the C-blocking around the wing.

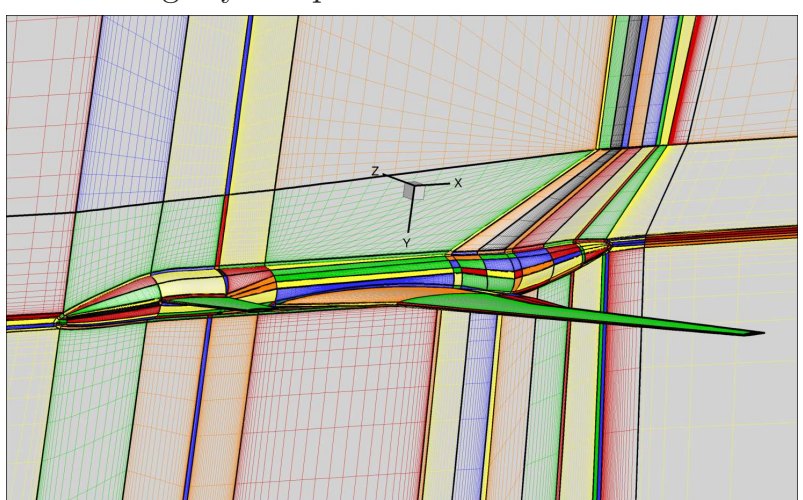

(a) Overall view of the grid model

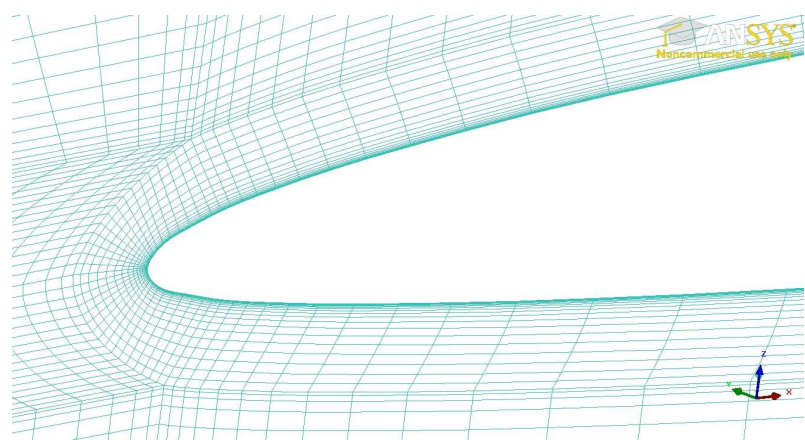

(b) View of a chordwise grid section on the LE of the wing

Figure 3. TCR grid model

Experimental investigations of the aerodynamic characteristics at low speed and up to high angles of attack were performed in the wind tunnel facilities at the Central Aerohydrodynamic Institute, TsAGI. The $\mathrm{T}-103$ wind tunnel is used for unsteady aerodynamic characteristics in the low subsonic velocity range. The wind tunnel has an open jet working section, of the continuous type, with an elliptical cross section 4.0 x $2.33 \mathrm{~m}$. Several configurations of the wind tunnel model were investigated to allow consideration of the influence of single components (vertical tail and canard wing) on the overall performances. Experimental tests were selected to provide knowledge of the following aspects 
1. static aerodynamic characteristics

2. rotary and unsteady aerodynamic derivatives

3. unsteady non-linear aerodynamic characteristics during large amplitude pitch oscillations

The normal and lateral forces and the moment coefficients from static and large amplitude pitch oscillations were measured. The mean values, in-phase and out-of-phase components of the force and moment coefficients were measured from the rotary and oscillatory motions. The tests were run at a value of free-stream speed of $40 \mathrm{~m} / \mathrm{s}$, which corresponds at sea level to a Mach number of 0.117 , and a Reynolds number of 0.778 million based on the mean aerodynamic chord of the wind tunnel model. The model centre of gravity was located along the fuselage centre line at $54.78 \%$ of the total length from the foremost point. The moment reference point and the centre of oscillatory motion coincide with this point. The main geometrical dimensions are reported in Table 2 .

\begin{tabular}{ll}
\hline \hline Reference wing area, $S$ & $0.3056 \mathrm{~m}^{2}$ \\
Reference wing span, $b$ & $1.12 \mathrm{~m}$ \\
Mean aerodynamic chord, $\bar{c}$ & $0.2943 \mathrm{~m}$ \\
Fuselage total length & $1.596 \mathrm{~m}$ \\
\hline \hline
\end{tabular}

Table 2. Reference values of the TCR wind tunnel model

\section{Numerical Results}

\section{A. Standard Dynamic Model Aircraft}

Pitching oscillatory motions were analyzed and various combinations of solver parameters examined to find those needed for a numerically well converged solution. The variation of the dynamic derivatives with Mach number, mean angle of incidence, reduced frequency and amplitude of applied motion were investigated. The test cases and corresponding flow conditions are summarized in Table 3. Each test case focuses on the effect of one motion parameter, as highlighted in the second line of the table.

\begin{tabular}{lllll} 
& Test Case 1 & Test Case 2 & Test Case 3 & Test Case 4 \\
\hline & $M$ & $\alpha_{0}$ & $k$ & $\alpha_{A}$ \\
\hline \hline Mach number, $M$ & 0.4 to 1.1 & 0.3 & 0.3 & 0.3 \\
Mean incidence, $\alpha_{0}$ & $0.0^{\circ}$ & 0.0 to $25.0^{\circ}$ & 0.0 to $25.0^{\circ}$ & 0.0 to $25.0^{\circ}$ \\
Reduced frequency, $k$ & 0.0493 & 0.0493 & 0.0493 to 0.2000 & 0.0493 to 0.0900 \\
Pitch amplitude, $\alpha_{A}$ & $5.0^{\circ}$ & $5.0^{\circ}$ & $5.0^{\circ}$ & 2.0 to $5.0^{\circ}$ \\
\hline \hline
\end{tabular}

Table 3. Description of the SDM test cases

\section{The Effect of Mach Number}

The influence of the Mach number on the damping derivative values through the transonic regime was examined on the coarse grid. The Mach number varied between 0.4 up to 1.1 with a finer step increment near Mach 1 where a significant change in damping value was observed. The reduced frequency was constant, so that an increment in the freestream speed was followed by a proportional increment in the frequency of motion. With a flowfield featuring the formation of shock waves and their time-dependent motion, a time step study was undertaken to evaluate the influence of the time resolution on the flow solution. Two sets of unsteady calculations were performed. First, the numerical solution was computed at 20 time steps uniformly distributed in one harmonic period and 3 cycles were simulated. Then, the number of time steps per cycle was increased to 80 , retaining the same number of cycles. Tests verified that an adequate convergence of the 
pseudo iterations was achieved. The influence of the time step is shown in Fig. 4, and shows that 20 time steps per pitching cycle are adequate for these cases.

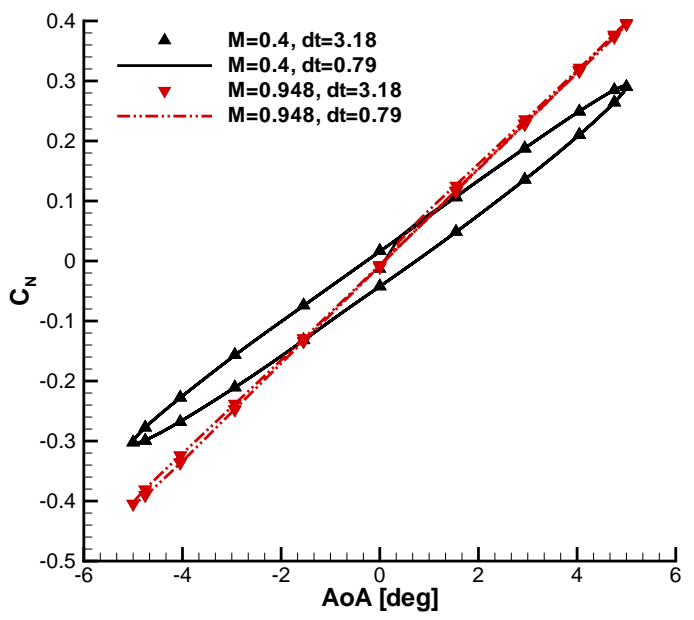

(a) Normal force coefficient

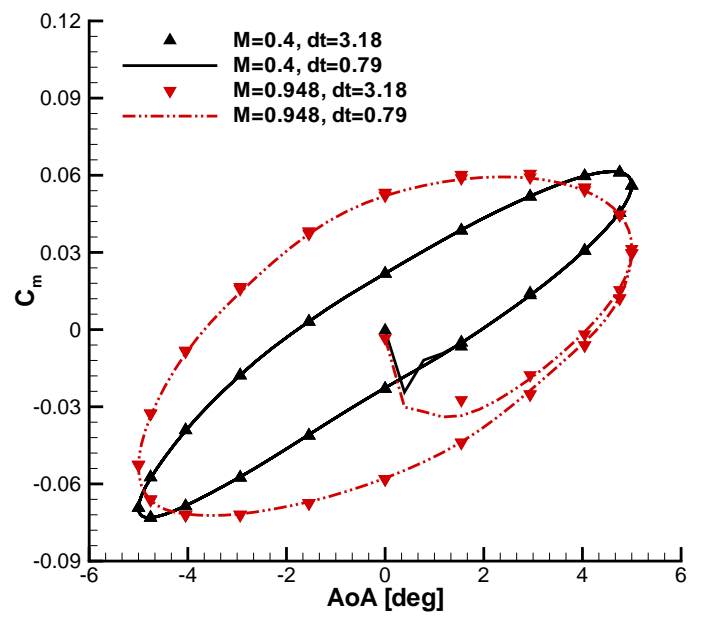

(b) Pitching moment coefficient

Figure 4. Test Case 1: normal force and pitching moment coefficient loops for the SDM model geometry using $20(\mathrm{dt}=3.18)$ and $80(\mathrm{dt}=0.79)$ time steps per pitching cycle

The variation of the damping-in-pitch derivative with Mach number is shown in Fig. 5. The predicted values using 20 and 80 time steps per cycle show an excellent agreement with each other. Wind tunnel and range data of Winchenbach et al. ${ }^{40}$ are included for comparison. The agreement of the present numerical results with experimental data is good. An increase in the damping is predicted for increasing Mach numbers, with the largest values in the transonic range near Mach 1. Beyond the transonic dip, which is slightly underpredicted, the numerical results predict well the reduction of the damping value.

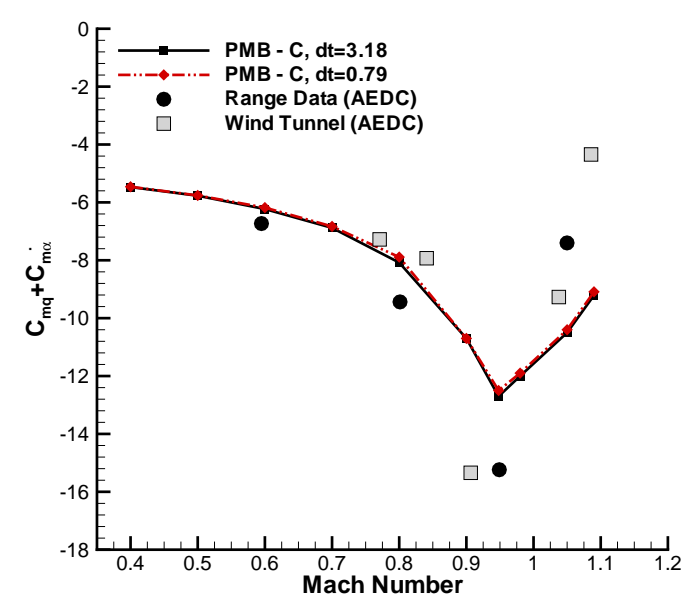

Figure 5. Test Case 1: influence of Mach number on the pitch damping derivative for the SDM model geometry

The mean value and the first harmonic of the surface pressure coefficient are shown in Fig. 6 for two values of Mach number. Variations of the pressure coefficient near the wing leading-edge are observed at Mach 0.4 and are in phase with the applied sinusoidal motion. The response of the horizontal tailplane in the pressure distribution has a phase lag of approximately $90^{\circ}$. The periodic movement of the shock wave over the wing surface at Mach number 0.948 has a phase lag with respect to the applied motion, as 
demonstrated by the real and imaginary parts of the pressure coefficient distribution over the main lifting surface. During the upstroke motion, the shock wave near the leading edge bends toward the wing tip and its downstream location moves till $70-80 \%$ of the tip chord. The moderate wing sweep is responsible for the shock wave advancing and, eventually, interacting with the shock wave at the trailing edge. A similar pattern is observed on the lower side of the wing surface for decreasing angle. Moving from the wing tip inward, the strength of the shock wave forming at the leading edge is reduced while an increase in strength is observed for the shock wave generating around the trailing edge. At Mach 0.948, the low pressure region on the horizontal tailplane reaches its maximum downstream location up to about $50 \%$ of the local chord. The horizontal tailplane provides the largest contribution to the damping-in-pitch derivative because of its moment arm from the centre of gravity. However, at this flow condition, the wing pressure distribution, as shown in Fig. 6(e), might provide an additional contribution to the damping value.

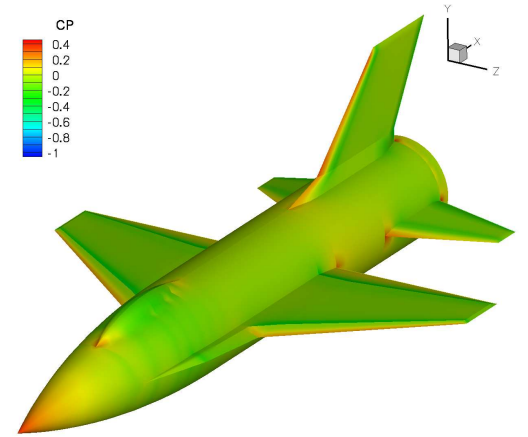

(a) Mean value, $M=0.4$

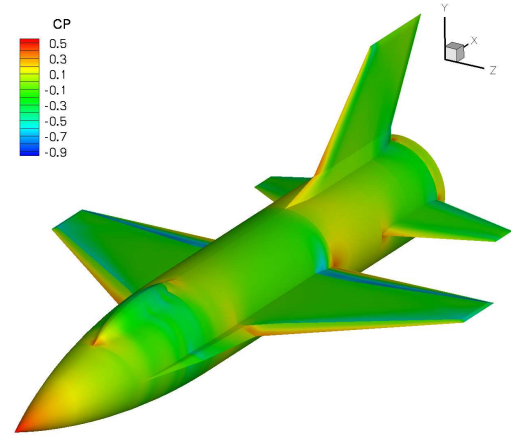

(d) Mean value, $M=0.948$

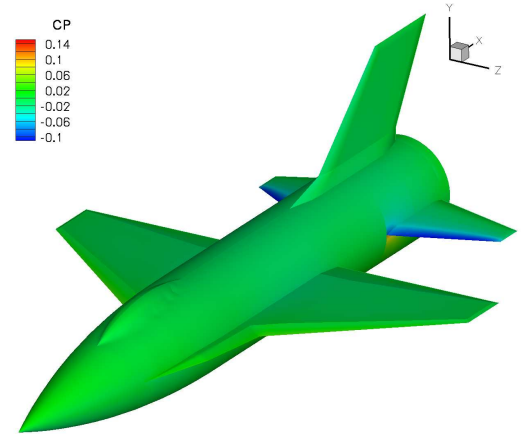

(b) Real part of $1^{\text {st }}$ harmonic, $M=0.4$

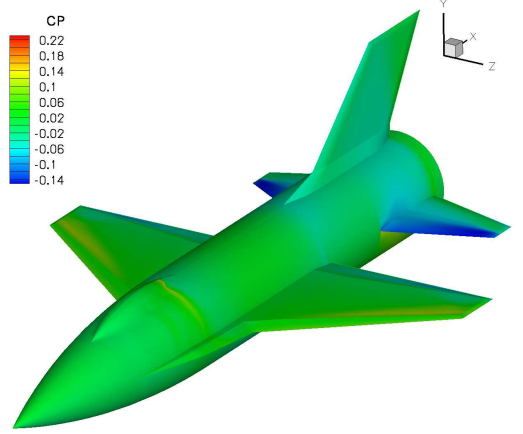

(e) Real part of $1^{\text {st }}$ harmonic, $M=0.948$

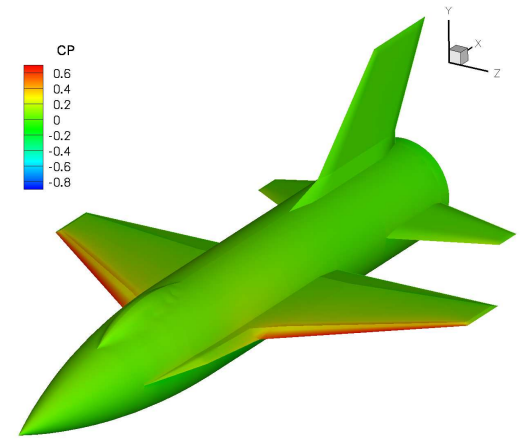

(c) Imaginary part of $1^{\text {st }}$ harmonic, $M=$ 0.4

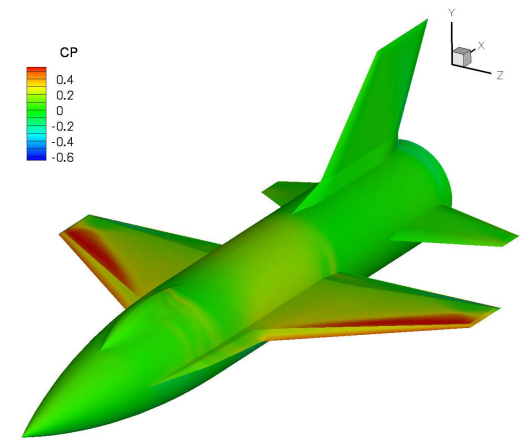

(f) Imaginary part of $1^{\text {st }}$ harmonic, $M=$ 0.948

Figure 6. Test Case 1: pressure coefficient distribution for the SDM model geometry for Mach number 0.4 and 0.948

\section{The Effect of Mean Angle of Incidence}

A number of calculations were made to investigate the influence of the mean angle of incidence, $\alpha_{0}$, on the normal force and pitching moment coefficient damping derivatives at low speeds. The mean angle of incidence varies between $0.0^{\circ}$ and $25.0^{\circ}$, with an increment of $5.0^{\circ}$. The $5.0^{\circ}$ mean angle of attack was not simulated because it is considered that an interpolation of the solutions at $\alpha_{0}=0.0^{\circ}$ and $10.0^{\circ}$ can provide a good approximation to the linear aerodynamics.

Fig. 7 shows the comparison of the CFD based damping derivatives with wind tunnel data at low speeds. The experimental data for the normal force damping derivative were measured in the continuous, open circuit trisonic wind tunnel of the aerodynamic lab at IHU, Iran. ${ }^{37}$ The pitch damping derivatives were measured in the low speed, closed circuit Ankara wind tunnel. ${ }^{36}$ Reasonable agreement is obtained between the two grids. The solutions using 40 and 80 steps per cycle yield very similar results. The magnitude of the peaks in the force and moment coefficients are mesh dependent, which is not unexpected for Euler solutions which 
rely on numerical diffusion in the region of the leading edge for the generation of vorticity. It is noted that the pitch damping derivatives continuously decrease as the angle of attack is increased, a dynamically stable condition. Beyond an angle of attack of $15^{\circ}$, the reduction of dynamic stability is due to the breakdown location of the strake vortices decreasing the longitudinal stability.

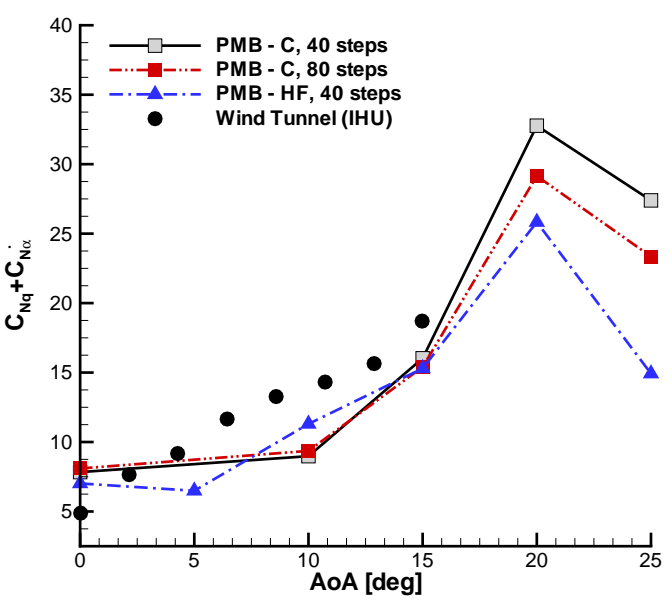

(a) Normal force coefficient damping

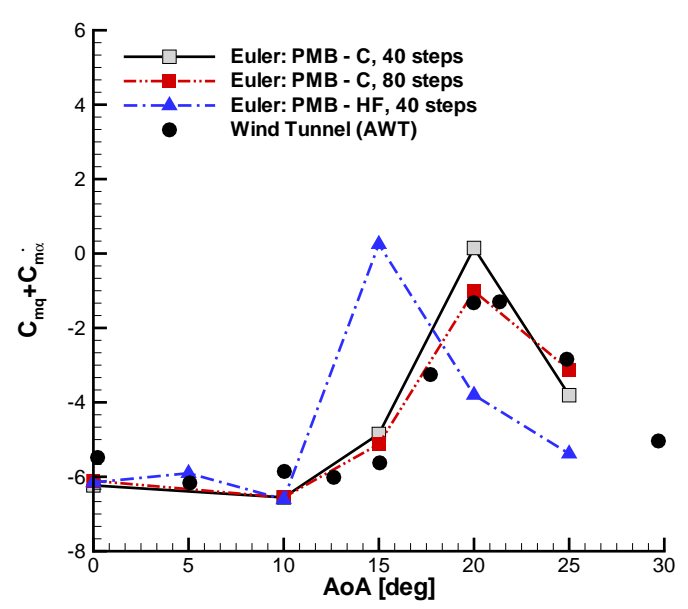

(b) Pitching moment coefficient damping

Figure 7. Test Case 2: influence of mean angle of attack on the damping derivatives for the SDM model geometry at Mach number 0.3

The time-histories of the longitudinal normal force and pitch moment coefficients at the mean angles of attack $0.0^{\circ}$ and $15.0^{\circ}$ are illustrated in Fig. 8 for the coarse grid using 80 steps per cycle. The variable along the horizontal axis is the increment of the instantaneous angle of attack during the applied motion with respect to the mean value, $\alpha_{0}$. All cycles of the unsteady calculations are included in the time-histories, indicated by Time Domain. The values of $C_{N}$ and $C_{m}$ at any instantaneous angle of attack between the upstroke and downstroke motion are different, indicating a time lag in the flowfield over the model. The difference between the motion of the model and the corresponding flowfield around it at any instantaneous angle of attack creates the hysteresis loops in both the simulated data. The effect of the mean angle of attack on the longitudinal coefficients of the SDM is clear. As the mean angle of attack increases from $0.0^{\circ}$ to $15.0^{\circ}$, the variations on the aerodynamic loads become non-linear. This indicates the formation of strake vortices, their breakdown and flow separation over the main lifting surfaces. Although $C_{N}$ is affected by these nonlinearities and/or flow separation, the main deviations are observed for the moment coefficient. The width and slope of the hysteresis loop for $C_{m}$ changes significantly and a characteristic figure of eight shape appears at the highest instantaneous angles of attack during the applied motion. The reconstructed aerodynamic coefficients using the fundamental harmonic, corresponding to the value of reduced frequency $k=0.0493$, are included and indicated by 1 Harmonic. At the lowest mean angle of attack, the reconstruction of the aerodynamic coefficients provides a fairly good approximation of the time-histories. With non-linearities occurring in the flow field at $\alpha_{0}=15.0^{\circ}$, only a partial representation of the time-histories is shown by the one-harmonic coefficients.

As expected in this aerodynamic state, an analysis of the frequency content of the aerodynamic coefficients reveals that the frequency spectrum contains several higher harmonics. In Fig. 9, the frequency spectrum of the normal force and pitching moment coefficient is shown. The magnitude of the harmonics decays exponentially with frequency and is insignificant for both $C_{N}$ and $C_{m}$ at higher frequencies. The magnitude of the second harmonic component at $0.0^{\circ}$ mean angle of attack is less than $10 \%$ of the magnitude of the fundamental harmonic. At $15.0^{\circ}$ mean angle of attack, the magnitude of the fourth harmonic component is less than $10 \%$ of the magnitude of the fundamental harmonic. The second and third harmonic frequencies contribute with around $50 \%$ and $20 \%$, respectively, to the content of the time-history. Considering the frequency spectrum of the time-histories, the reconstructed signal with only the fundamental harmonic, as illustrated in Fig. 8, lacks important information. 


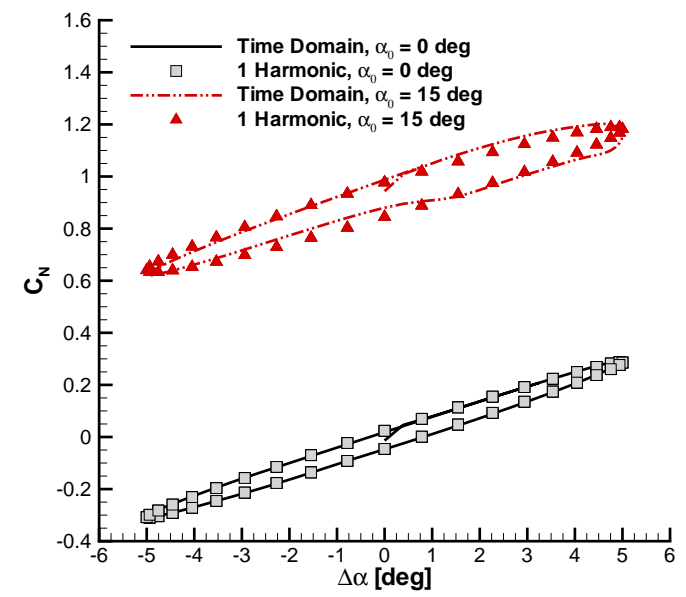

(a) Normal force coefficient

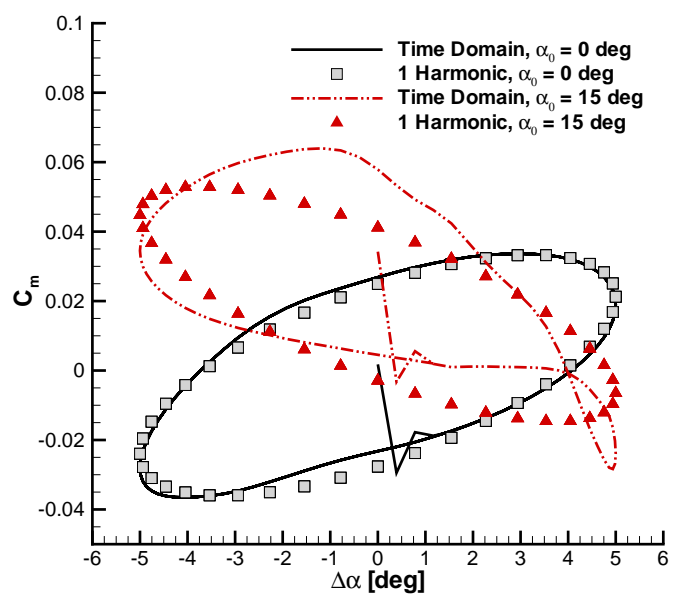

(b) Pitching moment coefficient

Figure 8. Test Case 2: normal force and pitch moment coefficients loops for the SDM geometry model for the coarse grid using 80 steps per cycle at two values of the mean angle of attack, $\alpha_{0}$

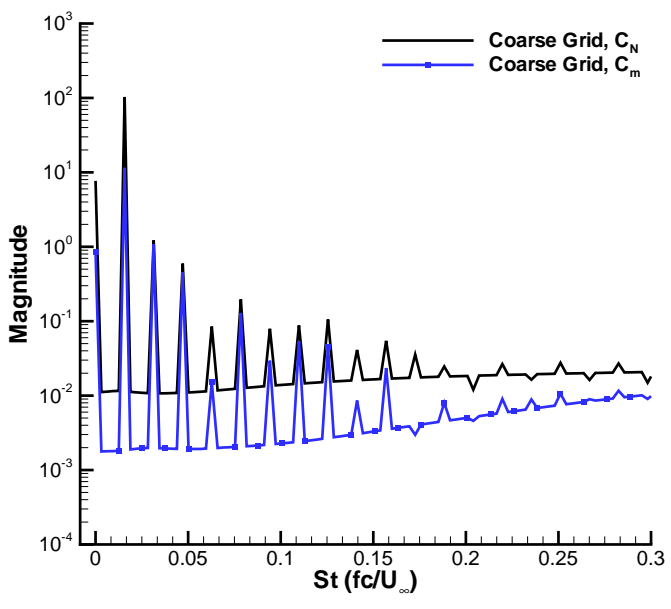

(a) Mean angle of attack, $\alpha_{0}=0.0^{\circ}$

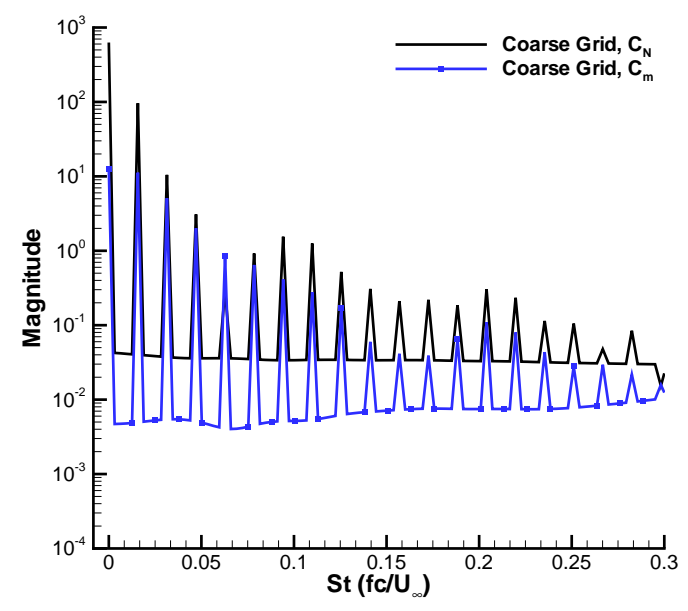

(b) Mean angle of attack, $\alpha_{0}=15.0^{\circ}$

Figure 9. Test Case 2: magnitude of normal force and pitching moment coefficients for SDM geometry model for the coarse grid using 80 steps per cycle

\section{The Effect of Reduced Frequency}

The influence of the reduced frequency on the dynamic derivatives was investigated at different values of mean angle of attack. The results included are for a Mach number of 0.3. The coarse grid is used in the present investigations and the solution for 40 steps per cycle included. Five cycles were simulated by the time-domain solver to achieve a steady harmonic output of the aerodynamic coefficients. The numerical technique for the extraction of damping derivatives was applied to the time-histories for the normal force and pitching moment coefficients. The dynamic derivative values, as function of the mean angle of attack, were computed for a data set of reduced frequencies. Four values of reduced frequency were selected: $\mathrm{k}=$ $0.0370,0.0493,0.0900$ and 0.2000 . The first three values are of the same order of magnitude of the reduced frequency usually applied in dynamic wind tunnel testing. These motions will be indicated in the following as slow motions, as opposed to rapid motion referring to the highest value of $k$. The computed dynamic 
derivative results are therefore expected to reasonably match the experimental data, which have already been used in the present validation. The highest value of reduced frequency is considered to evaluate the effect of a very rapid manoeuvre on the aircraft response, although the question if such a manoeuvre is practical is not considered.

Fig. 10 shows the comparison of computed dynamic derivatives with experimental measurements for a set of reduced frequencies. Results which were obtained from the simulations are similar at low angles of attack for both normal force and pitch moment coefficients.

It is considered, based on the presented data, a reasonable approximation to neglect the influence of the frequency of motion on the aerodynamic characteristics at low mean angles of attack. With aerodynamic non-linearities occurring in the flowfield at higher angles of attack, the effect of the reduced frequency has to be evaluated. Results for the slower motions, with frequency up to 0.0900, present a similar trend and a dip at around $20.0^{\circ}$ is clearly identified. Differences appear for the rapid motion, compared with experimental and numerical data, in the normal force damping derivatives. Though the damping values for the rapid motion show a dip at $20.0^{\circ}$ mean angle of attack, the value of the normal force dynamic derivatives is approximately half the value that was computed for the slower motions. A close match between slower and rapid motion is observed for the pitch moment dynamic derivatives.

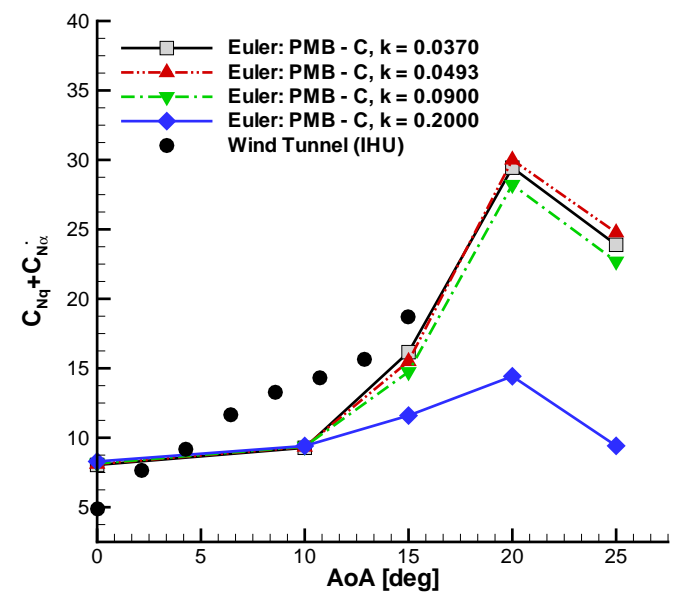

(a) Normal force coefficient damping

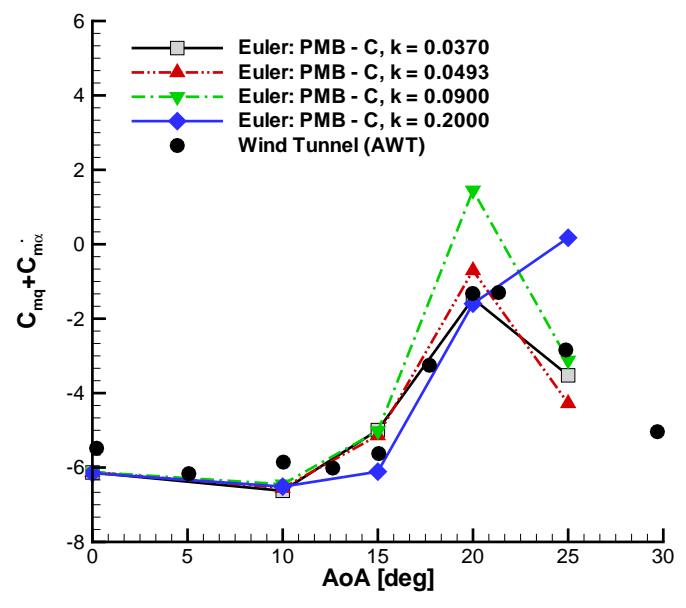

(b) Pitching moment coefficient damping

Figure 10. Test Case 3: influence of reduced frequency on the damping derivatives for the SDM geometry model at Mach number 0.3

Analysis of the time-history variation of the aerodynamic coefficients with the instantaneous angle of attack, for frequencies 0.0900 and 0.2000 , was considered at $15.0^{\circ}$ mean angle of attack. The loops of the aerodynamic loads for the slower and rapid motions, shown in Fig. 11, mostly differ at 2 points in one cycle of unsteadiness. This is observed when the instantaneous angle of attack is equal to the mean angle of motion during the upstroke and downstroke. The characteristic shape of eight in the pitch moment loop is only observed for motions at frequencies 0.0370 and 0.0493 . The motion at reduced frequency 0.0900 presents an inflection between an angle of attack of $15.0^{\circ}$ and $19.0^{\circ}$ which prevents the moment loop rolling into the shape of eight. This shape is lost in the rapid motion, resulting in the shape of an ellipse with its major axis aligned with the horizontal axis. The normal force loop is well approximated by an ellipse for the rapid maneuver. The loops for both aerodynamic coefficients obtained from the rapid motion enclose the loops from slower motions.

A similar conclusion about the influence of the reduced frequency of the applied motion on the stability dynamic derivatives can be drawn analysing the experimental dynamic measurements performed on the X31 A aircraft. ${ }^{26}$ The X-31 A is equipped with leading-edge flaps, trailing-edge flaperons and the horizontal stabilizer is replaced by a canard wing.

Investigations of the flow physics mechanisms responsible for strong frequency dependencies are undertaken at $15.0^{\circ}$ mean angle of attack. Analysis of the unsteady surface pressure distribution provided a preliminary although not conclusive explanation. Small changes in the mean value and imaginary compo- 


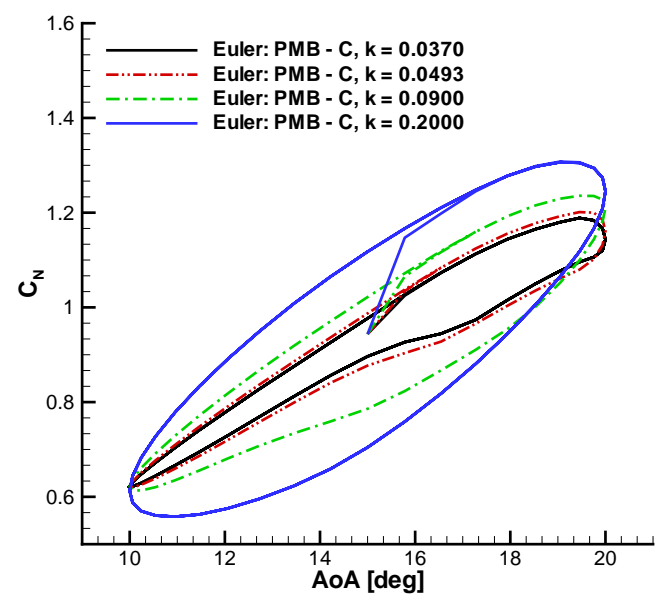

(a) Normal force coefficient

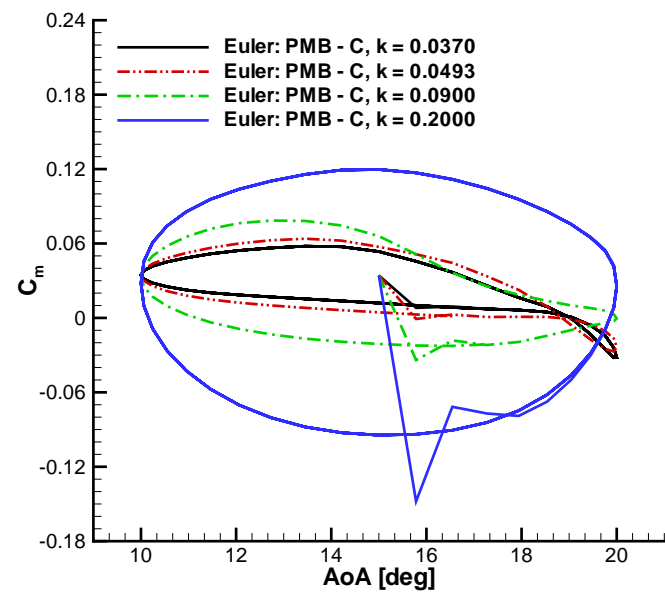

(b) Pitching moment coefficient

Figure 11. Test Case 3: normal force and pitch moment coefficients loops for the SDM geometry model at $15.0^{\circ}$ mean angle of attack for several reduced frequencies

nent of the fundamental frequency are observed in Fig. 12 for the pressure coefficient at the solid wall. The pressure scale and its distribution over the lifting surfaces have a noticeable variation. A larger suction region on the main wing is clearly responsible for the increase in the out of phase component for the force coefficient for a value of reduced frequency of 0.2000. A similar statement is valid for the hysteresis in the moment coefficient loop because of a large suction region at the leading edge of the tail plane. The mechanisms leading to changes in the pressure distribution are not clear yet.

\section{The Effect of Amplitude}

It is of interest to evaluate the influence that the amplitude of the sinusoidal motion have on the stability derivatives. The study was carried out for several values of the mean angle of attack and reduced frequencies. Three values of frequency are considered: 0.0370, 0.0493 and 0.0900. The amplitude of motion, $\alpha_{A}$, is $2.0^{\circ}$, $3.5^{\circ}$ and $5.0^{\circ}$ and the movement is applied through the centre of gravity. The results for the coarse and half-fine grids, using 40 steps per cycle, are presented in the following figures.

Fig. 13 shows the comparison of the dynamic derivative values for the normal force and pitch moment. At low mean angles of attack, the damping values computed for the coarse grid are very similar and distinguishing the results for different amplitudes is impossible. This is true for the three values of reduced frequency considered. Based on the presented data, it can be concluded that neglecting the influence of the amplitude of motion and its reduced frequency is a good approximation in the linear and quasi-linear aerodynamics, as expected from linearity. Analysing the time-histories of the aerodynamic coefficients at $0.0^{\circ}$ and $10.0^{\circ}$, the shape of the loops is not affected by the motion amplitude and a linear response is observed. In both $C_{N}$ and $C_{m}$, the time-history computed for the $3.5^{\circ}$ amplitude motion is half way between the $2.0^{\circ}$ and $5.0^{\circ}$ amplitude motions. With aerodynamic non-linearities occurring in the flow field, the influence of the amplitude of motion is evident for all frequencies of interest. Because experimental results were obtained simulating a $5.0^{\circ}$ amplitude motion, the values obtained from the smaller amplitude in general differ the most. Although for a moderate mean angle of attack the unsteadiness associated with the mean angle of attack might be insignificant, the superposition of the sinusoidal motion can introduce non-linearities at the higher instantaneous angles of attack.

To assess the prediction of dynamic stability derivatives, the half-fine grid was used in simulating a few test cases. The condition at $10.0^{\circ}$ mean angle of attack was considered. Test cases were run for the same values of reduced frequency and for amplitudes of motions of $2.0^{\circ}$ and $3.5^{\circ}$. Fig. 13 includes the results from the half-fine grid. Note that the half-fine grid-based damping derivatives well agree with the other numerical and experimental results. A perfect match is observed in the pitch damping derivatives for all considered 


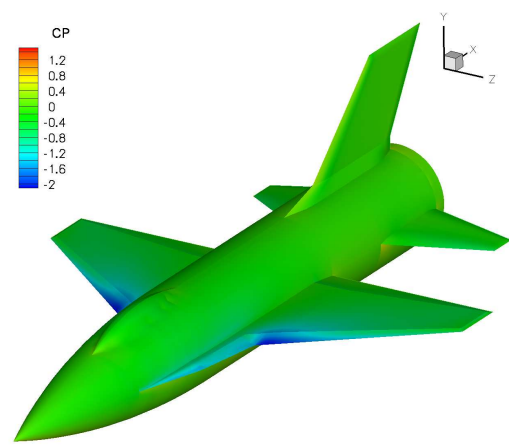

(a) Mean value, $k=0.09$

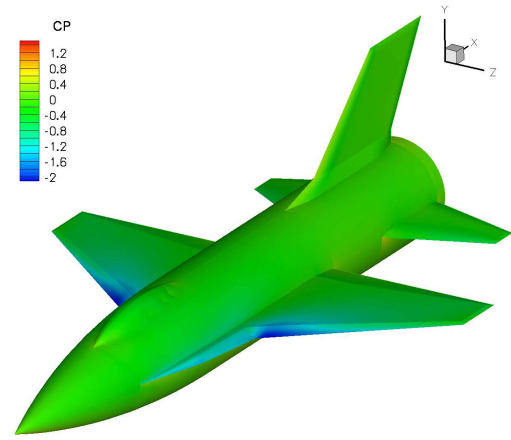

(d) Mean value, $k=0.20$

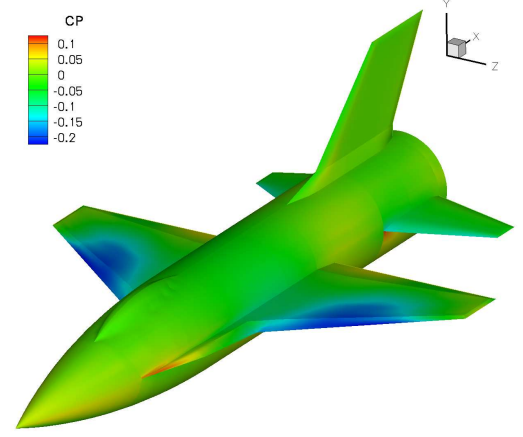

(b) Real part of $1^{\text {st }}$ harmonic, $k=0.09$

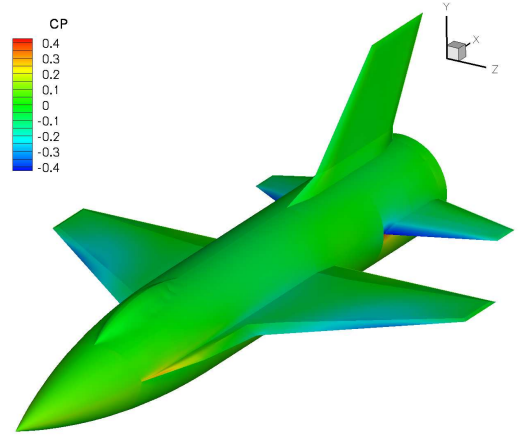

(e) Real part of $1^{\text {st }}$ harmonic, $k=0.20$

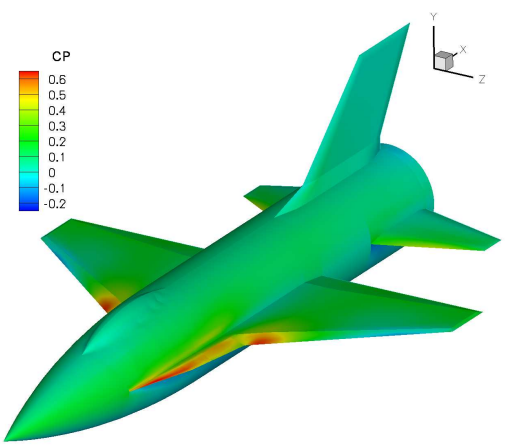

(c) Imaginary part of $1^{\text {st }}$ harmonic, $k=$ 0.09

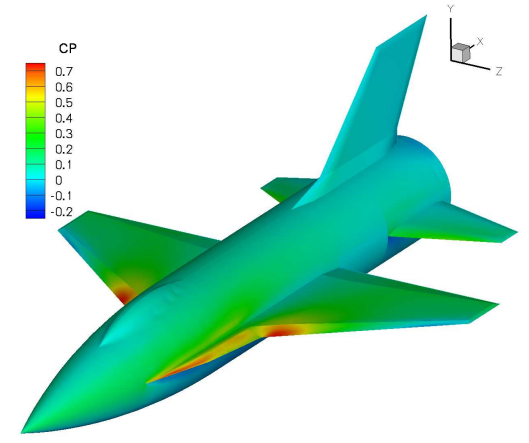

(f) Imaginary part of $1^{\text {st }}$ harmonic, $k=$ 0.20

Figure 12. Test Case 3: pressure coefficient distribution for the SDM model geometry at $15.0^{\circ}$ mean incidence for reduced frequency 0.09 and 0.20

flow conditions and without any dependency on the motion amplitude. A close match is also noted on the normal force damping derivatives. Results for the amplitude of motion of $2.0^{\circ}$ don't show any frequency dependence, as already pointed for the coarse grid. Results for $3.5^{\circ}$ amplitude of motion, although without any remarkable variations, are affected by the reduced frequency. With increase in the frequency of motion, a linear variation on the damping value is observed and at the higher reduced frequency a close match is presented for all numerical data.

\section{Study of Natural Unsteadiness}

Analysis of the natural unsteadiness of the flow at moderate angles of attack featuring vortical characteristics was undertaken for the coarse and half-fine grids. Several fixed geometry unsteady calculations were computed for a Mach number of 0.3 and angle of incidence of $10.0^{\circ}, 15.0^{\circ}$ and $20.0^{\circ}$. One of the most important factors in the execution of the unsteady calculation was the choice of the non-dimensional time step. The time step should be small enough to adequately resolve the unsteady fluctuations of the flow, but large enough not to increase excessively the required computational resources. Without any prior knowledge of the scale of the important frequencies in the flow, three values of the non-dimensional time step were selected equal to 0.01, 0.005 and 0.001 . The unsteady calculations used five thousand, ten thousand and fifty thousand real time steps for a non-dimensional duration time of 50, which corresponds to about 1.7 seconds based on the root chord and freestream speed.

Comparison of the normal force and pitching moment coefficients for the coarse and half-fine grids is shown in Fig. 14. The moment coefficient is taken at the centre of gravity. The fluctuations on both aerodynamic coefficients for the coarse grid unsteady calculations are insignificant and steady state values are obtained. The solutions using five thousand, ten thousand and fifty thousand real time steps were identical, so only one solution is included. Fluctuations on the aerodynamic loads around a mean value are observed for the half-fine grid calculations. The solutions for the three values of non-dimensional time 


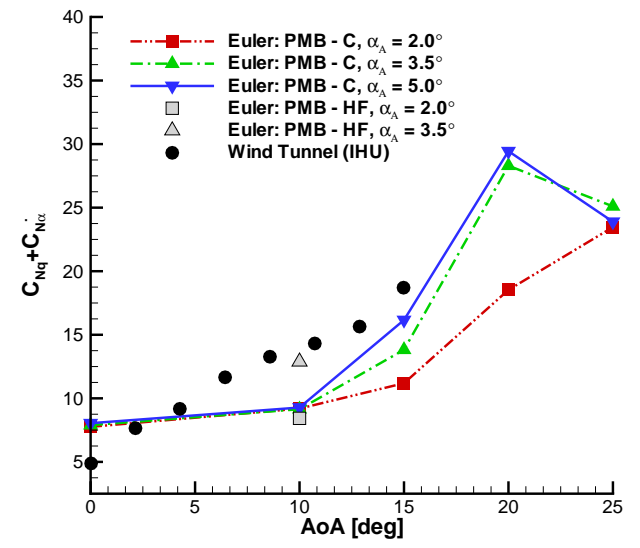

(a) Normal force coefficient, $k=0.0370$

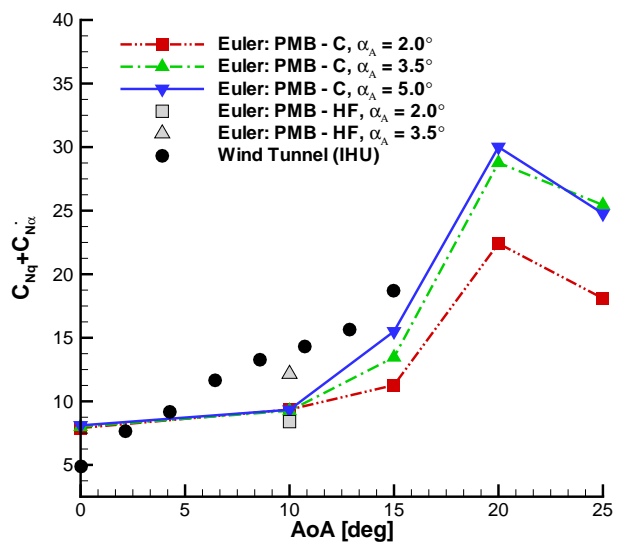

(c) Normal force coefficient, $k=0.0493$

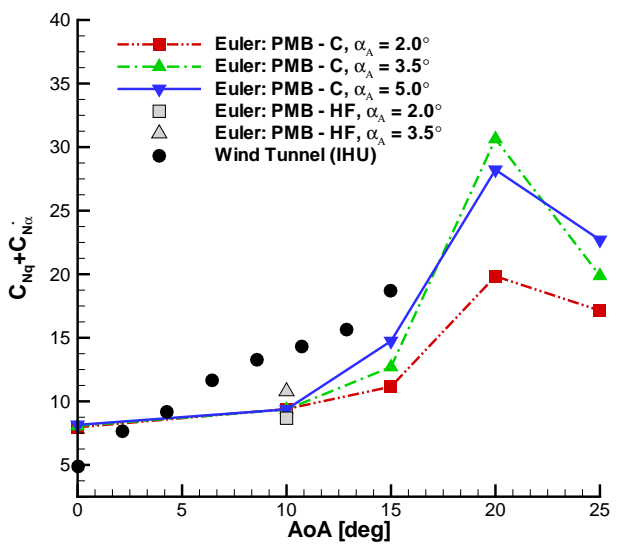

(e) Normal force coefficient, $k=0.0900$

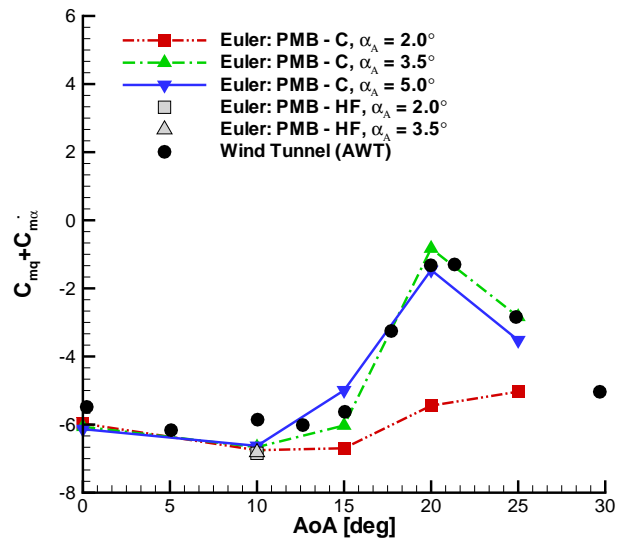

(b) Pitching moment coefficient, $k=0.0370$

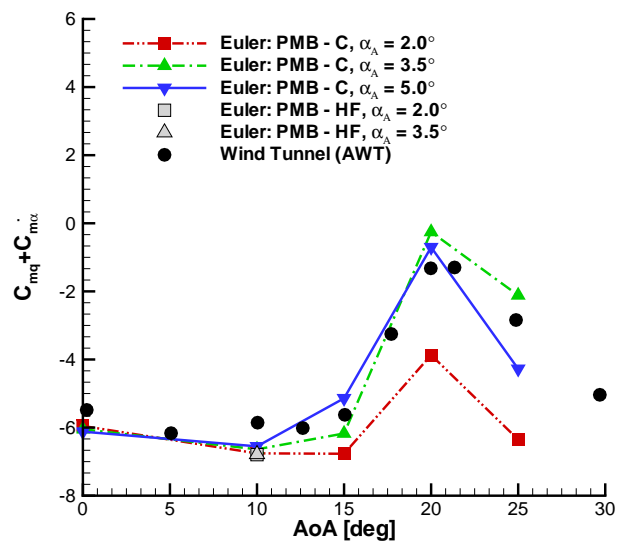

(d) Pitching moment coefficient, $k=0.0493$

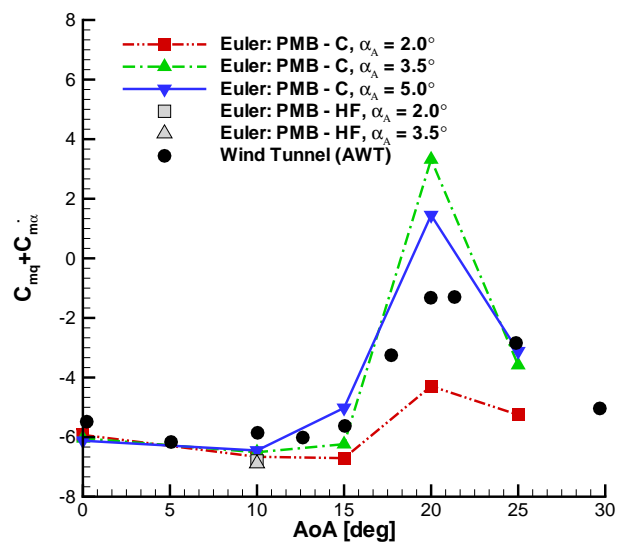

(f) Pitching moment coefficient, $k=0.0900$

Figure 13. Test Case 4: influence of amplitude on the damping derivatives for the SDM geometry model at Mach number 0.3 and several values of reduced frequency 
step are nearly identical at $10.0^{\circ}$ and $15.0^{\circ}$ incidence. At $20.0^{\circ}$, the unsteady solution using the largest non-dimensional time step agrees well with the solutions using smaller non-dimensional time steps.

Spectral analyses of the time signals are under investigation to shed some light on the source of unsteadiness observed for the half-fine grid.

\section{B. Transonic CRuiser Aircraft}

The prediction of aerodynamic characteristics of the TCR wind tunnel model includes both static and unsteady investigations, primarily at similar flow conditions to those obtained in the experimental measurements. First, validation of the static aerodynamic characteristics is examined. Then, numerical results for unsteady aerodynamics are presented.

The calculations of the TCR wind tunnel model were made on HECToR, ${ }^{41}$ the UK National Supercomputing Service, using 64 processors. The main difficulty with the calculations was the small CourantFriedrichs-Lewy (CFL) number required to avoid divergence. The current calculations were run at a CFL number of 4 . To obtain a well converged solution, the norm of the maximum residual and the integrated aerodynamic coefficients were monitored. Results for steady state analyses were run for four thousand iterations. At the highest angles of attack, above an incidence of $30^{\circ}$, the aerodynamic coefficients did not converge to an asymptotic value but presented undamped fluctuations around a mean value. In these circumstances, the mean value of the aerodynamic coefficients at the last ten thousand iterations was considered for the comparison of static aerodynamic characteristics. For forced pitching motions, the solution was computed at 200 time steps uniformly distributed in one period and 3 cycles considered. The numerical setup allowed to get a well converged solution in all test cases, featuring small and large pitching amplitudes. Fixed-geometry unsteady calculations were run to comprehend the natural unsteadiness of the flow and, in particular, to compare the solution with that from steady state analysis. A non-dimensional time step increment of 0.005 was considered and the non-dimensional final time set to 50, which corresponds to 0.37 seconds based on the wing aerodynamic chord and freestream speed.

\section{Validation of Static Aerodynamic Characteristics}

Predictions of static longitudinal aerodynamic characteristics were first validated for the TCR wind tunnel model. The model includes the vertical tail and the undeflected canard wing, with a symmetrical flow for all cases. A Mach number of 0.117 and a Reynolds number of 0.778 million, based on the wing mean aerodynamic chord and freestream speed, were used. Flow conditions are summarized in Table 4.

The low Reynolds number at the operating wind tunnel conditions and the leading-edge geometry of the TCR wind tunnel model are both critical factors to the CFD for the prediction of the initial flow separation, which affects the structure of the airflow around the scaled model and the overall measured aircraft performance. No transition tripping was installed in the wind tunnel model to trip the boundary layer to turbulent flow. Leading-edges of the TCR wind tunnel model raise the problem of predicting separation point around a smooth body shape. All simulations assumed fully turbulent flow.

\begin{tabular}{ll}
\hline \hline Freestream speed, $U_{\infty}$ & $40 \mathrm{~m} / \mathrm{s}$ \\
Reynolds number, $R e$ & $0.77810^{6}$ \\
Angle of incidence, $\alpha$ & $-10.0^{\circ}$ up to $40.0^{\circ}$ \\
Angle of sideslip, $\beta$ & $0.0^{\circ}$ \\
\hline \hline
\end{tabular}

Table 4. Description of the TCR test cases for static aerodynamic characteristics

Longitudinal force and moment coefficients for well converged calculations and experimental measurements are shown in Fig. 15. A close match is observed at negative up to moderate angles of incidence when vortical structures are not fully developed. With a complex system of vortices forming at higher incidences and massive flow separation, the deviations from experimental data are more significant. A parallel study ${ }^{42}$ includes more details on the break points in the pitching moment curve. The first break in the moment curve at around $5^{\circ}$ is due to a change coming from the wing which in turn seems to be caused by a change of the slope of the lift of the wing. A plateau in the moment coefficient follows the break point up to about $20^{\circ}$ and a mild reduction in the moment magnitude is observed in both data sets. The second break at around 


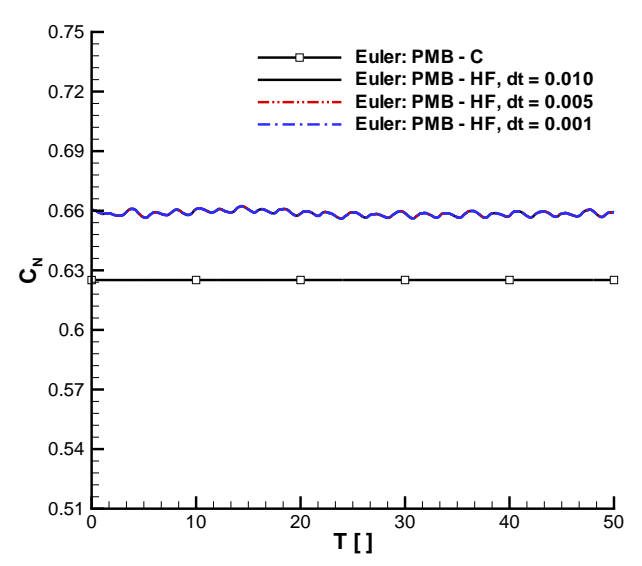

(a) Normal force coefficient, $\alpha=10.0^{\circ}$

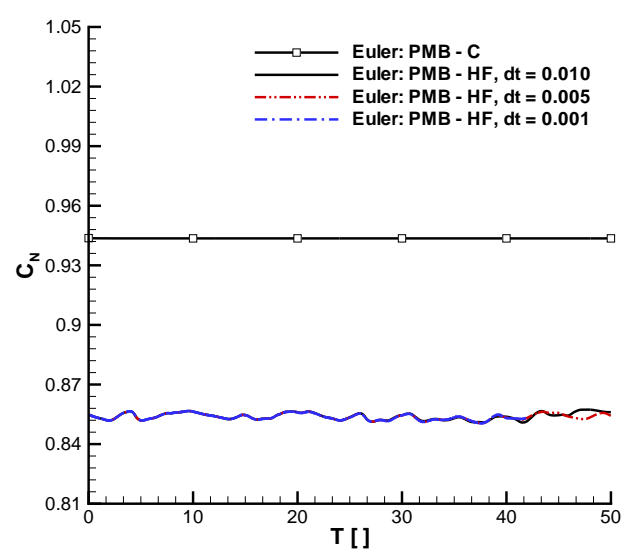

(c) Normal force coefficient, $\alpha=15.0^{\circ}$

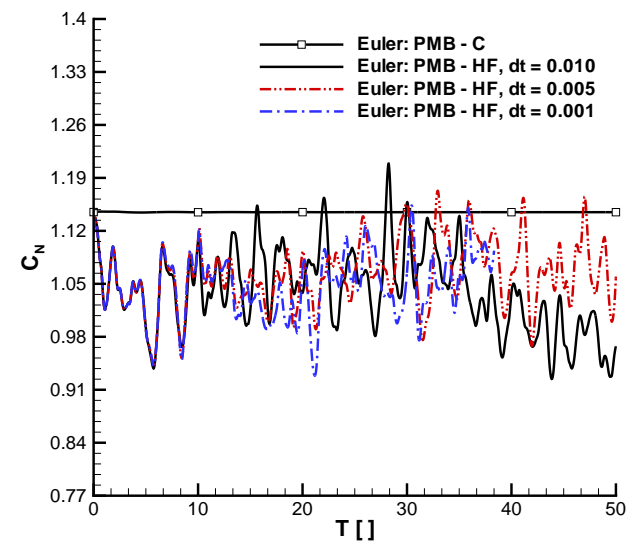

(e) Normal force coefficient, $\alpha=20.0^{\circ}$

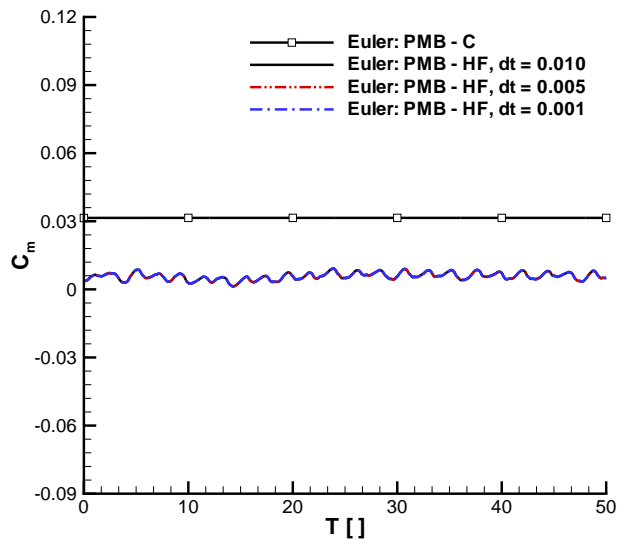

(b) Pitching moment coefficient, $\alpha=10.0^{\circ}$

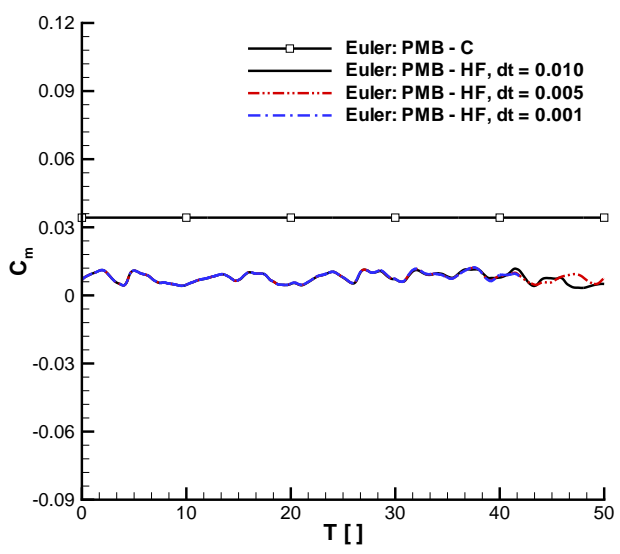

(d) Pitching moment coefficient, $\alpha=15.0^{\circ}$

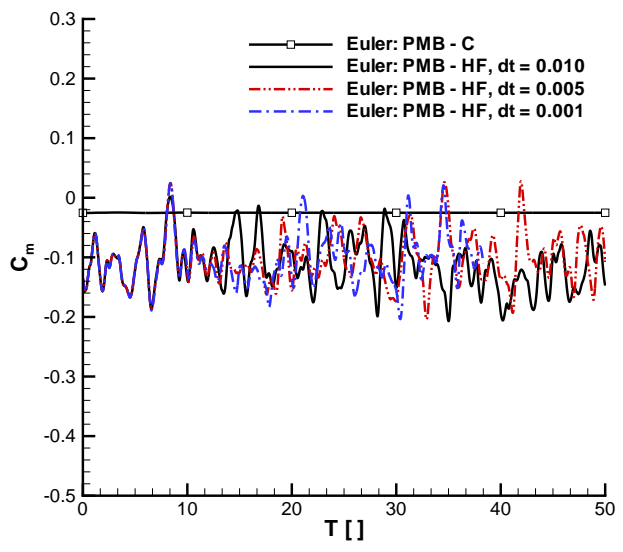

(f) Pitching moment coefficient, $\alpha=20.0^{\circ}$

Figure 14. SDM fixed geometry unsteady calculations for a Mach number of 0.3 
$20^{\circ}$ is possibly originated from the canard wing following the onset of the flow separation. Numerical results predict the first break in the moment curve, whilst delaying the second break point.

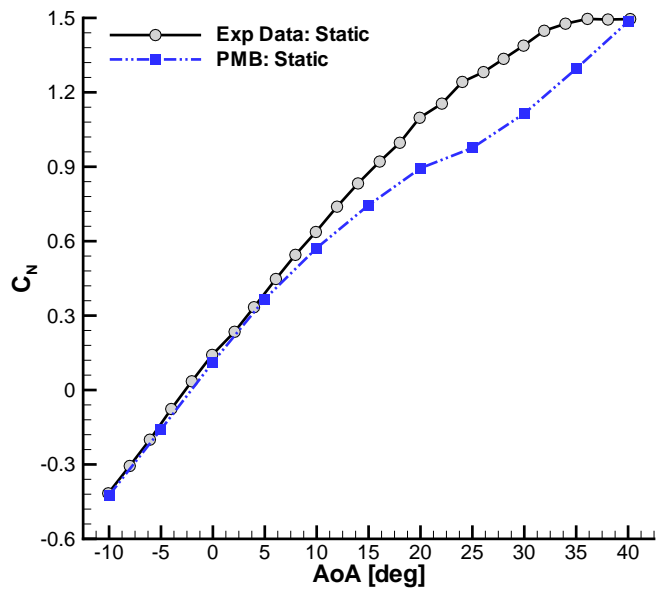

(a) Normal force coefficient

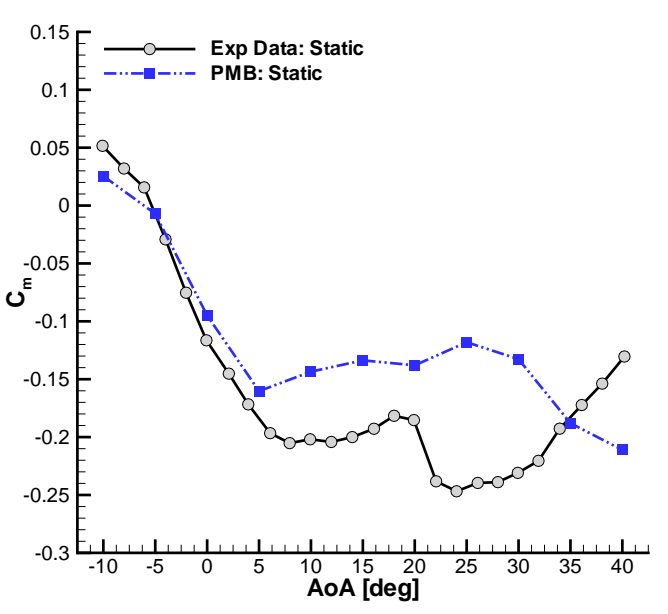

(b) Pitching moment coefficient

Figure 15. Static longitudinal aerodynamic characteristics for TCR wind tunnel model

Fig. 16 shows the pressure distribution with surface streamline patterns at several angles of attack. At $5^{\circ}$, the outer wing vortex originating from the wing leading-edge outboard of the crank and the canard vortex are observed. Primary reattachment lines are clearly visible in Fig. 16(a). The strength of the secondary vortex is very weak and the secondary separation and reattachment lines are close to the primary separation line for both wing and canard vortices. Smooth streamlines are predicted around the fuselage and in the inner wing section. Increasing the angle of incidence above the first break point, the inner wing vortex from the wing leading-edge inboard of the crank is observed. The streamline pattern suggests that the inner and outer wing vortices interact with each other over the main wing and a canard vortex is forming at its tip. The complexity of the flow structure is shown when the incidence is further increased through the second break point and, in particular, singular points in the velocity field indicate flow separation. Separation and reattachment lines are clearly visible. With the onset of canard separation, the flow around the fuselage is no longer well streamlined. More insights on the flow characteristics are found in a companion paper. ${ }^{42}$

\section{Unsteady Aerodynamic Derivatives for Pitch Oscillations}

Stability derivatives of the TCR wind tunnel model were obtained in the experimental campaign performing small amplitude forced oscillations. Numerical results restricted the forced motions around the three body axes to pitch oscillation only. The rotation point is at the centre of gravity in all test cases. Similar flow conditions to those considered in the static investigations were used for the Mach and Reynolds numbers. Motion parameters are summarized in Table 5. The reduced frequency, based on freestream speed and wing mean aerodynamic chord, is 0.0234 .

\begin{tabular}{ll}
\hline \hline Freestream speed, $U_{\infty}$ & $40 \mathrm{~m} / \mathrm{s}$ \\
Mean incidence, $\alpha_{0}$ & $0.0^{\circ}$ up to $30.0^{\circ}$ \\
Pitch amplitude, $\alpha_{A}$ & $3.0^{\circ}$ \\
Frequency, $f$ & $1.0 \mathrm{~Hz}$ \\
\hline \hline
\end{tabular}

Table 5. Description of TCR test cases for small amplitude pitch oscillations

Normal force and pitching moment damping derivatives are compared in Fig. 17 to experimental results for several mean angles of attack. Prediction of damping values is in agreement with measured data. For 


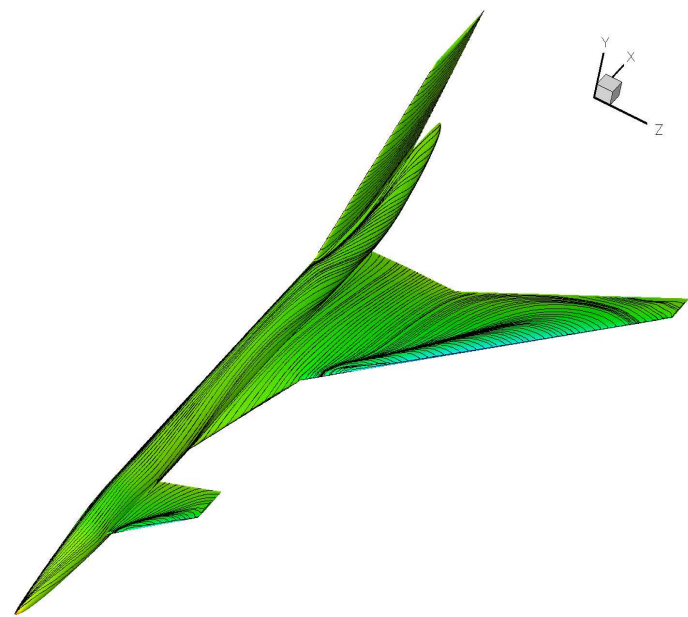

(a) $\alpha=5^{\circ}$

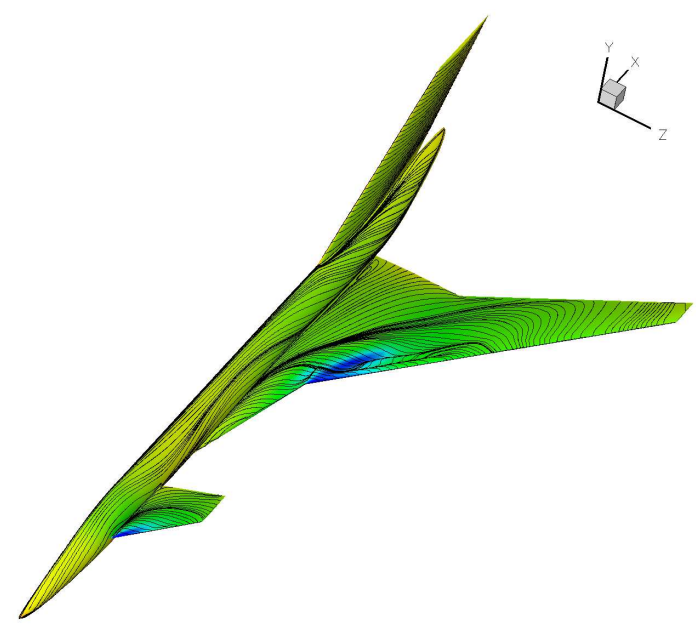

(c) $\alpha=15^{\circ}$

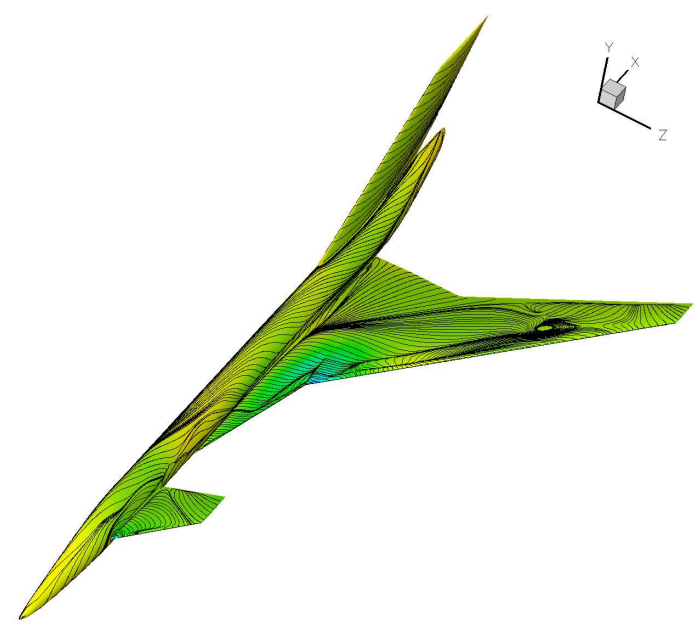

(e) $\alpha=25^{\circ}$

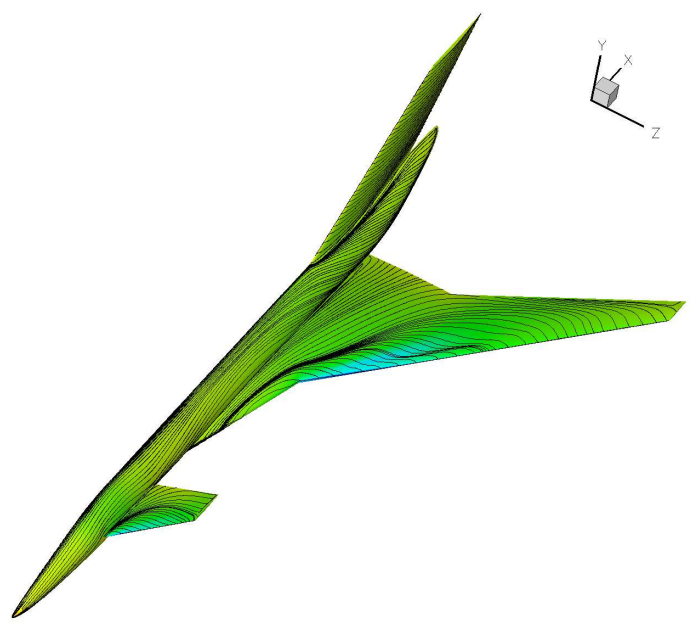

(b) $\alpha=10^{\circ}$

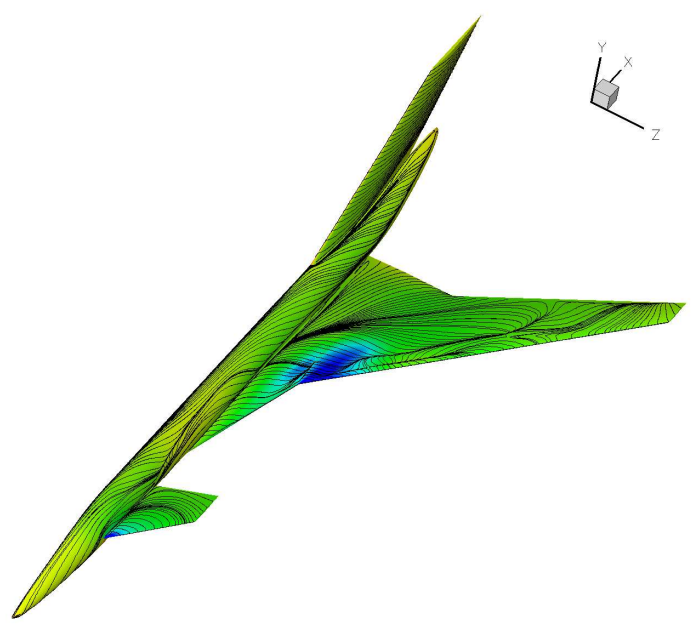

(d) $\alpha=20^{\circ}$

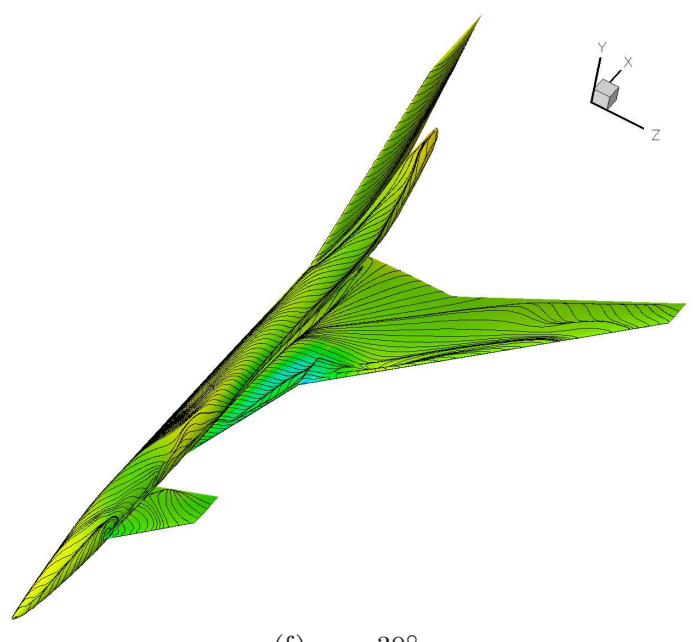

(f) $\alpha=30^{\circ}$

Figure 16. Pressure distribution with surface streamline pattern for TCR wind tunnel model at several angles of attack $\left(\beta=0.0^{\circ}, U_{\infty}=40 \mathrm{~m} / \mathrm{s}\right.$ and $\left.R e=0.77810^{6}\right)$

the force coefficient damping, the comparison is good for all cases up to large incidences. Experimental results show a positive damping in pitch at around $20^{\circ}$ mean angle of attack, with a noticeable frequency

\section{0 of 27}


dependence. Unstable characteristics in pitch were explained by massive canard flow separation. ${ }^{43}$ The prediction of pitch damping values fails to indicate this important feature at the mean angles identified by measurements. Nonetheless, positive damping is found in the numerical results at slightly larger incidences. Measurements during small amplitude forced motions did not include time-histories of aerodynamic coefficients, making more difficult to trace the origin of this delay. A first comment is that for the static curve of the moment coefficient, the second break occurs at an angle of attack similar to the mean angle of attack experiencing unstable characteristics during forced motion. Although no evidence has been given yet to prove this statement, it's our understanding to interpret the flow physics as the amenable mechanics to both situations. Numerical results predict a second break in the moment curve with a slight delay, of about $5^{\circ}$ to $10^{\circ}$, after the experimental break point, and at the same mean angles of attack anti-damping in pitch is observed. The same flow phenomena leading to the above connection between static and dynamic investigations is likely to be the driving mechanics in the numerical experiments. A final remark is for the relatively poor prediction of damping values, which are of larger magnitude than the experimental data, at $10^{\circ}$ mean angle of attack. Strong non-linear features are locally not experienced in both data sets and the plateaux in the moment curve are in agreement. Considering non-critical flow conditions, it is not clear yet the reason the numerical result overpredict the magnitude of the damping values.

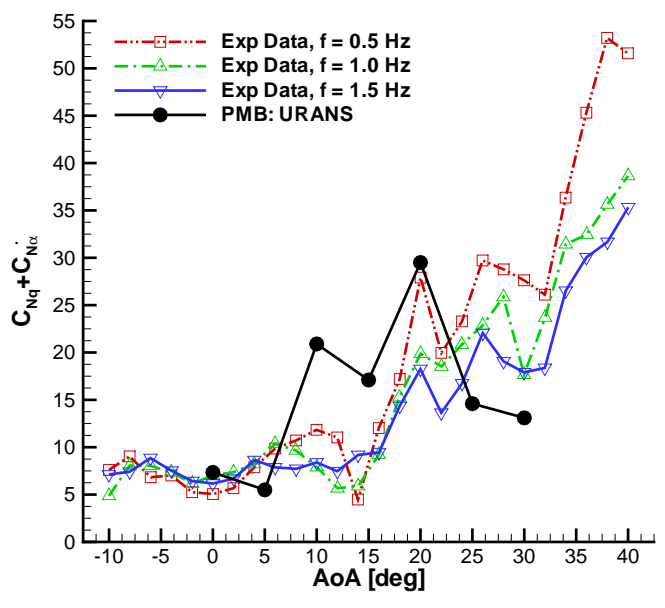

(a) Normal force coefficient damping

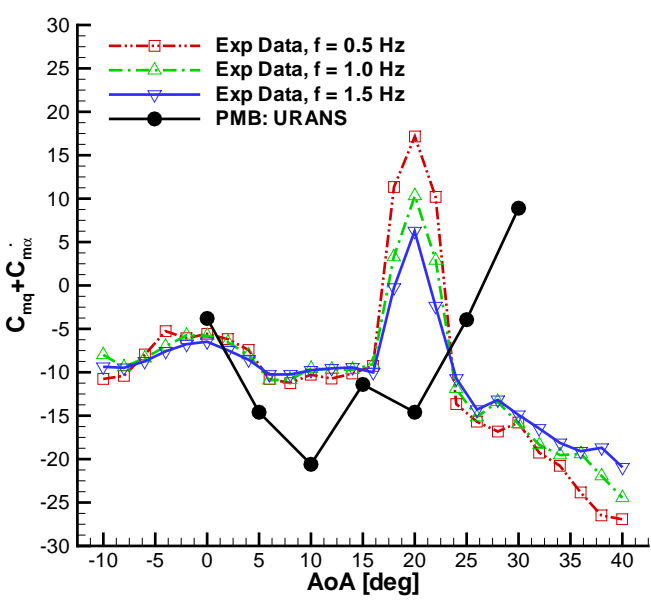

(b) Pitching moment coefficient damping

Figure 17. Dynamic derivatives for TCR wind tunnel model undergoing forced oscillations in pitch

The force and moment coefficient loops for the model undergoing forced oscillation in pitch axis are superimposed to the static curves in Fig. 18. Numerical solutions were well converged and only one cycle is included for the unsteady calculations. A favourable comparison is achieved between the two sets of calculations. At $5^{\circ}$ mean angle of attack, a significant hysteresis is predicted for the moment coefficient which may be caused by local flow features at the first break point in the pitching moment curve.

\section{Unsteady Non-Linear Aerodynamics during Large Amplitude Pitch Oscillations}

Forces and moments acting on a moving aerodynamic model at high angle of attack are non-linear functions of the motion variables. To achieve a deeper insight into the numerical simulations in the non-linear regime, several test cases were run for large amplitude pitch oscillations, for which time-histories of the measured aerodynamic coefficients are available.

The same frequency of motion of $1 \mathrm{~Hz}$ is considered for all test cases, which feature a mean angle of incidence of $8.0^{\circ}$ and $18.0^{\circ}$ and a pitch amplitude of $10.0^{\circ}$ and $20.0^{\circ}$. Table 6 summarizes the motion parameters.

Numerical results for the force and moment coefficient loops are compared against experimental results in Fig. 19 for Test Case 1 and Test Case 2. A favourable agreement is observed for both cases. When increasing the pitch amplitude from $10.0^{\circ}$ to $20.0^{\circ}$, the more significant impact is on the moment coefficient. Both numerical and experimental force coefficient loops have a slightly larger mean slopes than the corresponding 


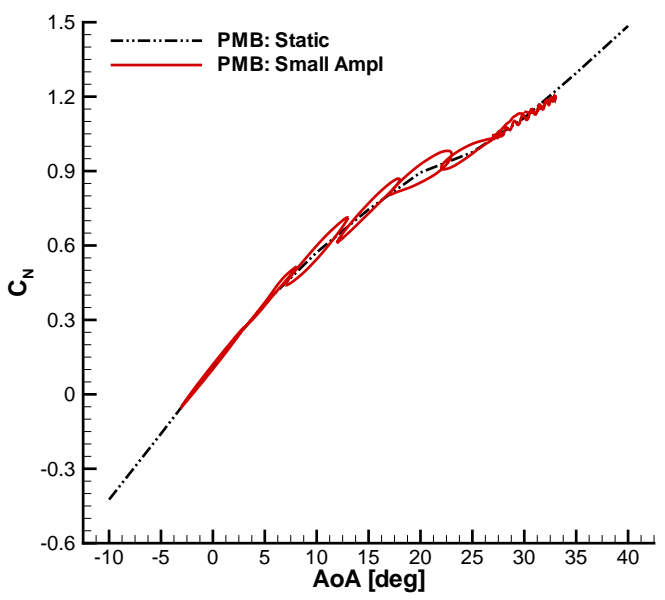

(a) Normal force coefficient

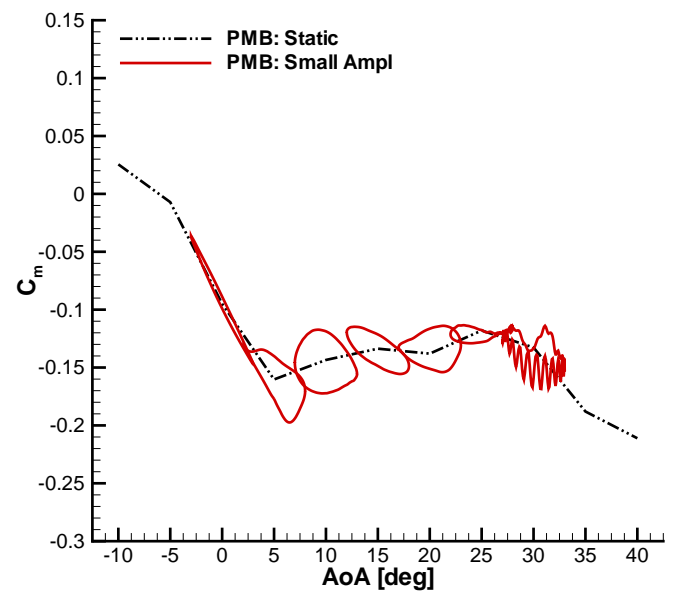

(b) Pitching moment coefficient

Figure 18. Normal force and pitching moment coefficient loops for the TCR wind tunnel model performing small amplitude pitch oscillations compared to static values

\begin{tabular}{lllll}
\hline & Test Case 1 & Test Case 2 & Test Case 3 & Test Case 4 \\
\hline \hline Freestream speed, $U_{\infty}$ & $40 \mathrm{~m} / \mathrm{s}$ & $40 \mathrm{~m} / \mathrm{s}$ & $40 \mathrm{~m} / \mathrm{s}$ & $40 \mathrm{~m} / \mathrm{s}$ \\
Mean incidence, $\alpha_{0}$ & $8.0^{\circ}$ & $8.0^{\circ}$ & $18.0^{\circ}$ & $18.0^{\circ}$ \\
Pitch amplitude, $\alpha_{A}$ & $10.0^{\circ}$ & $20.0^{\circ}$ & $10.0^{\circ}$ & $20.0^{\circ}$ \\
Frequency, $f$ & $1.0 \mathrm{~Hz}$ & $1.0 \mathrm{~Hz}$ & $1.0 \mathrm{~Hz}$ & $1.0 \mathrm{~Hz}$ \\
\hline \hline
\end{tabular}

Table 6. Description of TCR test cases for large amplitude pitch oscillations

static curves. Main features in the measured moment loops are also found in the numerical solutions. The numerical solutions differ the most from experimental data at the lowest angles of attack and, in particular, the shape of eight is observed in all cases.

Comparison for Test Case 3 and Test Case 4 is shown in Fig. 20. The overall agreement of numerical results with measurements is good for all cases. The pitch amplitude has a significant effect on the results. Whilst the two cases have the same mean angle of attack, an offset between numerical and experimental data is noted for a pitch amplitude of $10^{\circ}$. The solutions for Test Case 4 overlap the experimental results, while retaining most of the features in the experimental loops. The reason for obtaining a closer agreement to experimental data (not in terms of shape) for the larger amplitude pitch oscillations is not clear yet, although considering the influence of the second break point could shed some light on this aspect.

\section{Study of Natural Unsteadiness}

Fixed geometry unsteady calculations were run to investigate the natural unsteadiness of the flow at moderate to large angles of attack and compare the steady state predictions. Flow conditions are summarized in Table 7. The non-dimensional time step used in the calculations is 0.005 . The final non-dimensional time is 50, which corresponds to a physical time of 0.37 seconds based on the freestream velocity and wing aerodynamic chord.

Unsteady calculations were inizialized by a steady state solution obtained after ten thousand iterations. In Fig. 21, the normal force and pitching moment coefficients as function of the non-dimensional time are compared to static values for well converged steady analyses after four thousand iterations. An initial comment is that steady state results after ten thousand iterations are not well converged and only used for initialization, whilst the well converged steady values included in figure are in agreement with the unsteady 


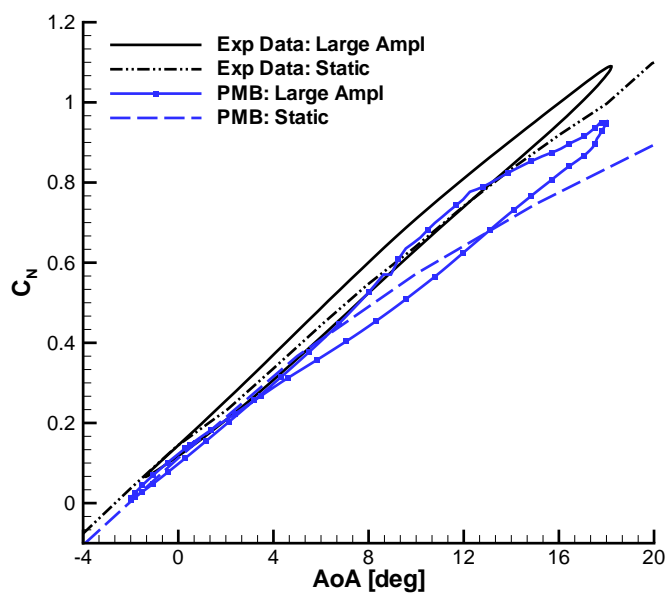

(a) Normal force coefficient, Test Case 1

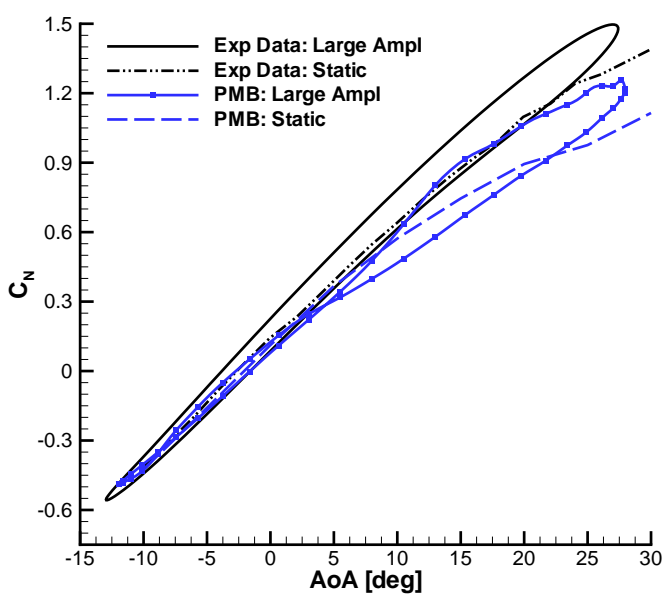

(c) Normal force coefficient, Test Case 2

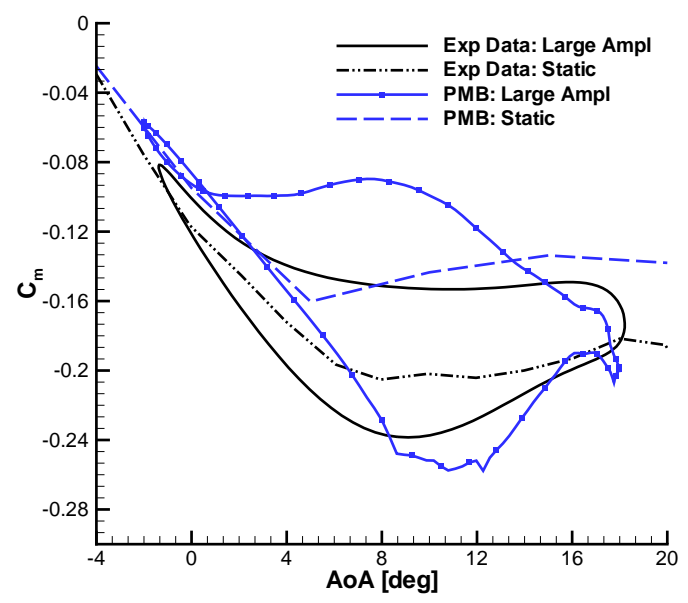

(b) Pitching moment coefficient, Test Case 1

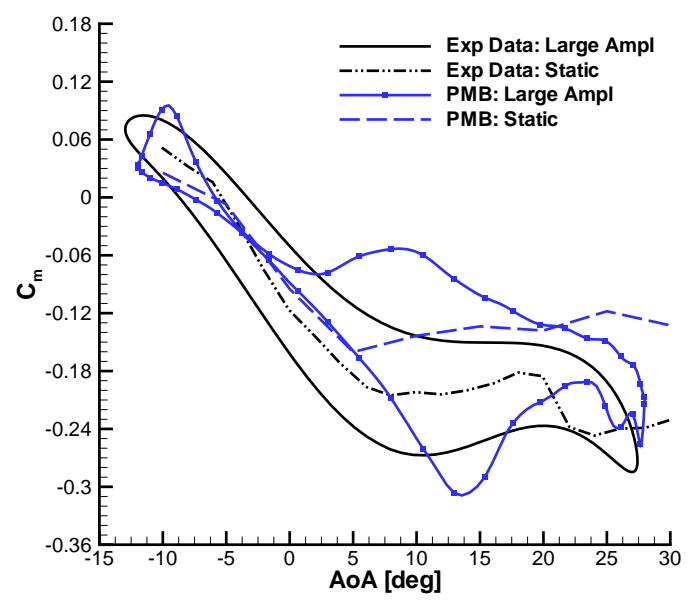

(d) Pitching moment coefficient, Test Case 2

Figure 19. Normal force and pitching moment coefficient loops for TCR wind tunnel model for Test Case 1 $\left(\alpha_{0}=8^{\circ}, \alpha_{A}=10^{\circ}\right.$ and $\left.f=1 \mathrm{~Hz}\right)$ and Test Case $2\left(\alpha_{0}=8^{\circ}, \alpha_{A}=20^{\circ}\right.$ and $\left.f=1 \mathrm{~Hz}\right)$

\begin{tabular}{ll}
\hline \hline Freestream speed, $U_{\infty}$ & $40 \mathrm{~m} / \mathrm{s}$ \\
Angle of attack, $\alpha_{0}$ & $15.0^{\circ}$ up to $40.0^{\circ}$ \\
Non-dimensional time step, dt & 0.005 \\
Number of time steps & 10000 \\
\hline \hline
\end{tabular}

Table 7. Description of TCR test cases for fixed unsteady calculations

values. Convergence of the unsteady values to the well converged steady results indicates, first, the convergence of static calculations and, second, the lack of any unsteady effect. At large incidences above $30.0^{\circ}$, the unsteady values after an initial settlement deviate from the static results. A final remark is the presence of undamped oscillations in the convergence of aerodynamic coefficients for the steady state analyses and their poorer agreement with unsteady values is caused by unsteadiness in the flow structure. Although of moderate intensity, unsteady aerodynamic coefficients present fluctuations at the higher angles of attack. 


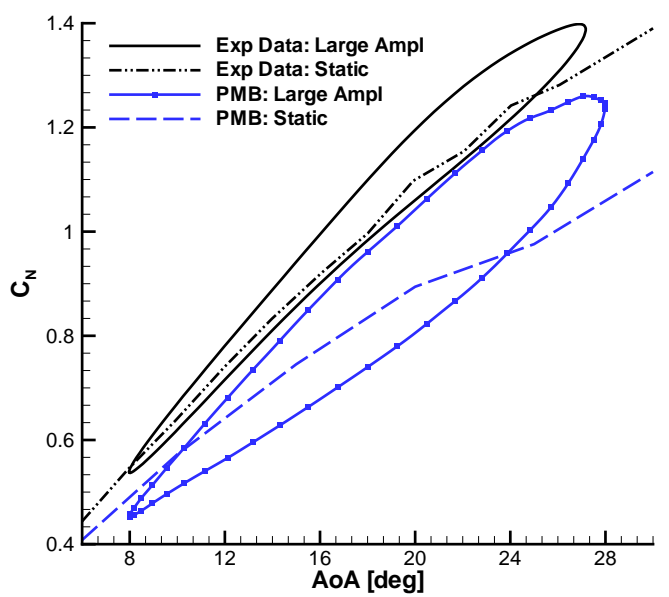

(a) Normal force coefficient, Test Case 3

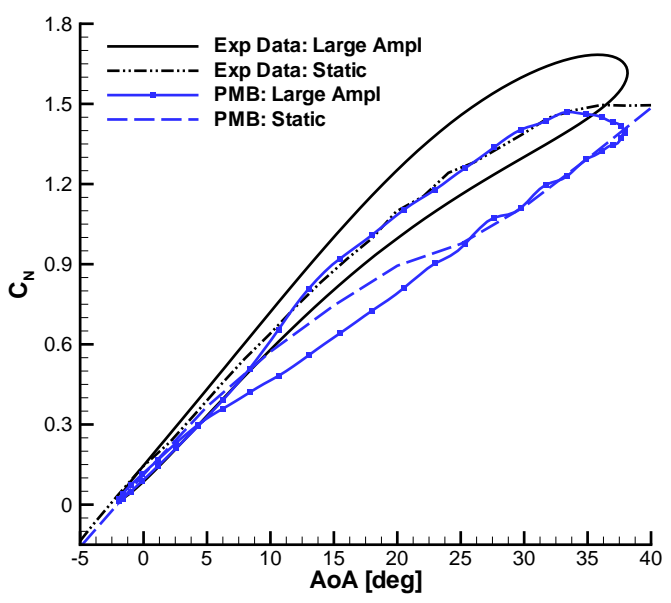

(c) Normal force coefficient, Test Case 4

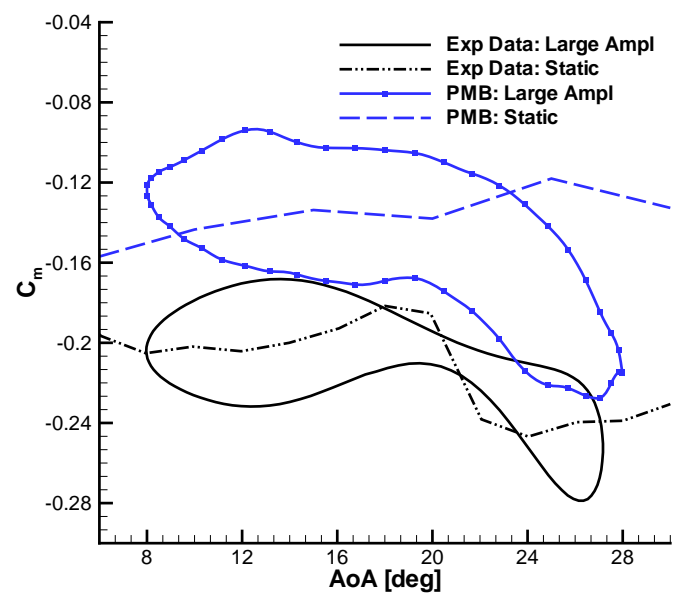

(b) Pitching moment coefficient, Test Case 3

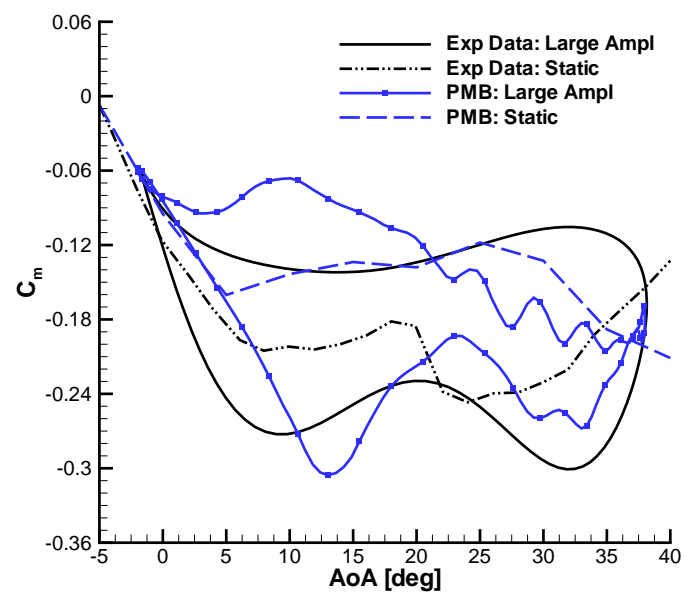

(d) Pitching moment coefficient, Test Case 4

Figure 20. Normal force and pitching moment coefficient loops for TCR wind tunnel model for Test Case 3 $\left(\alpha_{0}=18^{\circ}, \alpha_{A}=10^{\circ}\right.$ and $\left.f=1 \mathrm{~Hz}\right)$ and Test Case $4\left(\alpha_{0}=18^{\circ}, \alpha_{A}=20^{\circ}\right.$ and $\left.f=1 \mathrm{~Hz}\right)$

Experimental measurements for static investigations were averaged over two seconds but time histories were not recorded.

\section{Conclusions}

An unsteady time-accurate CFD solver is used for the computation of aerodynamic loads for forced periodic motions. The Standard Dynamic Model (SDM) and the Transonic CRuiser (TCR) wind tunnel geometries are the test cases. Two numerical techniques for the estimation of dynamic derivatives are implemented. The first is an accurate evaluation of the Fourier integral; the second is based on a linear regression mathematical model. Similar results were obtained. The flow is modelled using the Euler equations for the SDM and the RANS equations for the TCR. A good agreement with available experimental data is observed. A study for understanding the influence of motion parameters was also conducted for the SDM. For the TCR, the prediction of longitudinal aerodynamic characteristics is validated against experimental data at low speed and up to high angle of incidence. A benchmark against experimental data is presented for 
small and large amplitude forced oscillations in pitch. Prediction of dynamic derivatives was in agreement with wind tunnel data.

\section{Acknowledgments}

The first author gratefully acknowledgments the support by the SimSAC project under funding from the Sixth Framework programme of the European Union. Computer time was provided though the UK Applied Aerodynamics Consortium (UKAAC) under EPSRC grant EP/F005954/1.

\section{References}

${ }^{1}$ Orlik-Rückemann, K. J., "Dynamic Stability Testing of Aircraft-Needs versus Capabilities," Progress in Aerospace Sciences, Vol. 16, 1975, pp. 431-447.

${ }^{2}$ Bryan, G. H., "Stability in Aviation," MacMillan, London, 1911.

${ }^{3}$ Greenwell, D. I., "Frequency Effects on Dynamic Stability Derivatives Obtained from Small-Amplitude Oscillatory Testing," Journal of Aircraft, Vol. 35, No. 5, September-October, 1998, pp. 776-783.

${ }^{4}$ Nguyen, L. T., "Evaluation of Importance of Lateral Acceleration Derivatives in Extraction of Lateral-Directional Derivatives at high Angles of Attack," NASA TN D-7739, October, 1974.

${ }^{5}$ Wagner, H., "Üeber die Entstehung des dynamischen Auftriebes von Tragflügeln," Zeitschrift für angewandte Mathematik und Mechanik, Vol 1, Book 1, 1925, pp. 17-35.

${ }^{6}$ Küssner, H. G. K., "Stresses Produced in Airplane Wings by Gusts," NASA TM 654, January, 1932.

${ }^{7}$ Jones, R. T. and Fehlner, L. F., "Transient Effects of the Wing Wake on the Horizontal Tail," NACA TN 771, 1940.

${ }^{8}$ Tobak, M., "On the Use of the Indicial Function Concept in the Analysis of Unsteady Motions of Wings and Wing-Tail Combinations," NACA Report 1188, 1954.

${ }^{9}$ Tobak, M. and Schiff, L. B., "On the Formulation of the Aerodynamic Characteristics in Aircraft Dynamics," NACA TR $\mathrm{R}-456,1976$. 1966.

${ }^{10}$ Volterra, V., Theory of Functionals and of Integral and Integro-Differential Equations, Dover Pub., Inc., New York,

${ }^{11}$ Goman, M. G., Stolyarov, G. I., Tyrtyshnikov, S. L., Useltsev, S. P., and Khrabrov, A. N., "Mathematical Description of Aircraft Longitudinal Aerodynamic Characteristics at High Angle of Attack Accounting for Dynamic Effects of Separated Flow," TsAGI Preprint No. 9, 1990.

${ }^{12}$ Goman, M. G. and Khrabrov, A. N., "State-Space Representation of Aerodynamic Characteristics of an Aircraft at High Angles of Attack," Journal of Aircraft, Vol. 31, No. 5, September-October, 1994, pp. 1109-1115.

${ }^{13}$ Boelens, O. J., Badcock, K. J., Elmilgui, A., Abdol-Hamid, K. S., and Massay, S. J., "Comparison of Measured and Block Structured Simulation Results for the F-16XL Aircraft," Journal of Aircraft, Vol. 46, No. 2, 2009, pp. 377-384.

${ }^{14}$ Badcock, K. J., Richards, B. E., and Woodgate, M. A., "Elements of Computational Fluid Dynamics on Block Structured Grids using Implicit Solvers," Progress in Aerospace Sciences, Vol. 36, 2000, pp. 351-392.

${ }^{15}$ Anderson, J. D., Computational Fluid Dynamics - The basic with applications, Mechanical Engineering Series, McGrawHill International Editions, 1995.

${ }^{16}$ Osher, S. and Chakravarthy, S., "Upwind Schemes and Boundary Conditions with Applications to Euler Equations in General Geometries," Journal of Computational Physics, Vol. 50, No. 3, 1983, pp. 447-481.

${ }^{17}$ Van Leer, B., "Towards the Ultimate Conservative Difference Scheme II: Monotonicity and Conservation Combined in a Second Order Scheme," Journal of Computational Physics, Vol. 14, No. 4, 1974, pp. 361-374.

${ }^{18}$ Jameson, A., "Time Dependent Calculations Using Multigrid, with Applications to unsteady Flows Past Airfoils and Wings," AIAA Paper 91-1596, 1991.

${ }^{19}$ Spalart, P. R. and Allmaras, S. R., "A One Equation Turbulence Model for Aerodynamic Flows," In the 30th AIAA Aerospace Science Meeting and Exhibit, AIAA Paper 1992-0439, January 1992.

${ }^{20}$ Wilcox, D. C., "Reassesment of the Scale Determining Equation for Advanced Turbulence Models," AIAA Journal, Vol. 26, November, 1988, pp. 1299-1310.

${ }^{21}$ Brandsma, F. J., Kok, J. C., Dol, H. S., and Elsenaar, A., "Leading Edge Vortex Flow Computations and Comparison with DNW-HST Wind Tunnel Data," Proceeds of the RTO/AVT Symposium on Vortex Flows and High Angle of Attack, NATO RTO/AVT, 2001.

${ }^{22}$ Klein, V., Murphy, P. C., Curry, T. J., and Brandon, J., "Analysis of Wind Tunnel Longitudinal Static and Oscillatory Data of the F-16XL Aircraft," NASA TM-97-206276, December, 1997.

${ }^{23}$ Da Ronch, A., Ghoreyshi, M., Badcock, K. J., Görtz, S., Widhalm, M., Dwight, R. P., and Campobasso, M. S., "Linear Frequency Domain and Harmonic Balance Predictions of Dynamic Derivatives," 28th AIAA Applied Aerodynamic Conference, AIAA-2010-4699, Chicago, IL, 2010.

${ }^{24}$ Klein, V. and Noderer, K. D., "Modeling of Aircraft Unsteady Aerodynamic Characteristics. Part 2-Parameters Estimated From Wind Tunnel Data," NASA TM 110161, April, 1995.

${ }^{25}$ Klein, V. and Noderer, K. D., "Modeling of Aircraft Unsteady Aerodynamic Characteristics. Part 1-Postulated Models," NASA TM 109120, May, 1994.

${ }^{26}$ Smith, M. S., "Analysis of Wind Tunnel Oscillatory Data of the X-31A Aircraft," NASA CR 1999-20875, February, 1999.

${ }^{27}$ Morelli, E. A., "High Accuracy Evaluation of the Finite Fourier Transform using Sampled Data," NASA TM 110340 , 1997. 
${ }^{28}$ Klein, V., "Maximum Likelihood Method for Estimating Airplane Stability and Control Parameters from Flight Data in Frequency Domain," NASA TP 1637, May, 1980.

${ }^{29}$ Filon, L. N. G., "On a Quadrature Formula for Trigonometric Integrals," Proceedings of the Royal Society of Edinburgh, Vol. 49, 1928, pp. 38-47.

${ }^{30}$ Brigham, O. E., The Fast Fourier Transform, Prentice-Hall, Englewood Cliffs, N.J., 1974.

${ }^{31}$ Rabiner, L. R., Schafer, R. W., and Rader, C. M., "The Chirp-z Transform Algorithm and Its Application," The Bell System Technical Journal, May-June, 1969, pp. 1249-1292.

${ }^{32}$ Junkins, J. L. and Kim, Y., Introduction to Dynamics and Control of Flexible Structures, American Institute of Aeronautics and Astronautics, Inc., Washington, DC, AIAA Education Series, Blacksburg, Virginia, 1993.

${ }^{33}$ Huang, X. Z., "Wing and Fin Buffet on The Standard Dynamic Model," NATO RTO Report Number, RTO-TR-26, pp. 361-381.

${ }^{34}$ Beyers, M. E., "Subsonic Roll Oscillation Experiments on the Standard Dynamic Model," AIAA Atmospheric Flight Mechanics Conference, AIAA-83-2134, 1983.

${ }^{35}$ Jermey, C. and Schiff, L. B., "Wind Tunnel Investigation of the Aerodynamic Characteristics of the Standard Dynamic Model in Coning Motion at Mach 0.6," 12nd Atmospheric Flight Mechanics Conference, AIAA-85-1828, 1985.

${ }^{36}$ Alemdaroglu, N., Iyigun, I., Altun, M., Uysal, H., Quagliotti, F., and Guglieri, G., "Determination of Dynamic Stability Derivatives Using Forced Oscillation Technique," 40th Aerospace Science Meeting and Exhibition, AIAA-2002-0528, 2002.

${ }^{37}$ Davari, A. R. and Soltani, M. R., "Effects of Plunging Motion on Unsteady Aerodynamic Behaviour of an Aircraft Model in Compressible Flow," Iranian Journal of Science and Technology, Transaction B Enginneering, Vol. 31, No. B1, 2007, pp. 49-63.

${ }^{38}$ Von Kaenel, R., Rizzi, A., Oppelstrup, J., Grabowski, T., Ghoreyshi, M., Cavagna, L., and Bérard, A., "CEASIOM: Simulating Stability \& Control with CFD/CSM in Aircraft Conceptual Design," 26th International Congress of the Aeronautical Sciences, ICAS, 2008.

39 "ANSYS ICEM CFD," http://www.ansys.com/products/icemcfd.asp.

${ }^{40}$ Winchenbach, G. L., Uselton, B. L., Hathaway, W. H., and Chelekis, R. M., "Comparison of Free-Flight and Wind Tunnel Data for a Generic Fighter Configuration," AIAA Paper 82-1365, 1982.

41 "HECToR: UK National Supercomputing Service," http://www.hector.ac.uk/.

${ }^{42}$ Eliasson, P., Vos, J. B., Da Ronch, A., Mengmeng, Z., and Rizzi, A., "Virtual Aircraft Design of TransCRuiser Computing Break Points in Pitch Moment Curve," 28th AIAA Applied Aerodynamic Conference, AIAA-2010-4366, 2010.

${ }^{43}$ Khrabrov, A., Kolinko, K., Zhuk, A., and Grishin, I., "Wind Tunnel Test Report," SimSAC Report, Delivery Number D 6.4-7, March 2010. 


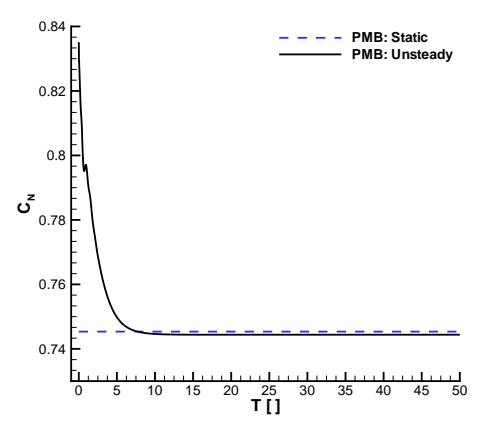

(a) Normal force coefficient, $\alpha=15.0^{\circ}$

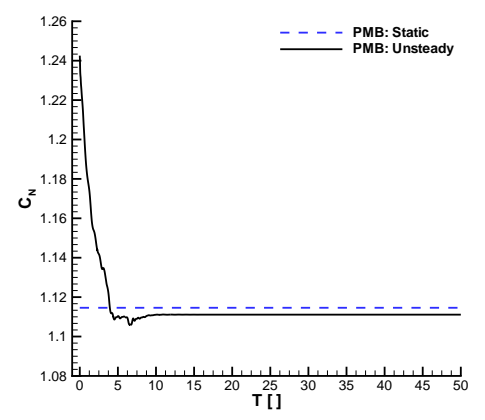

(d) Normal force coefficient, $\alpha=30.0^{\circ}$

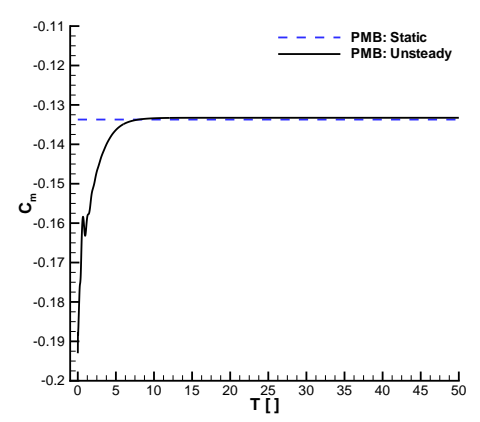

(g) Pitch moment coefficient, $\alpha=15.0^{\circ}$

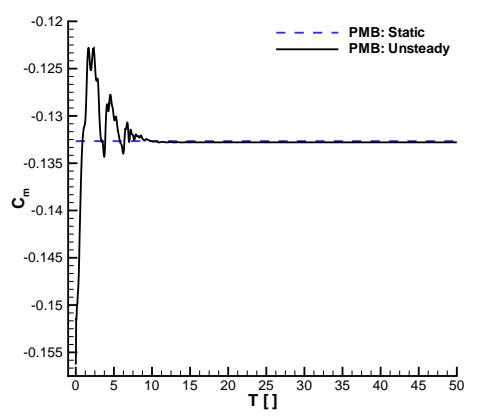

(j) Pitch moment coefficient, $\alpha=30.0^{\circ}$

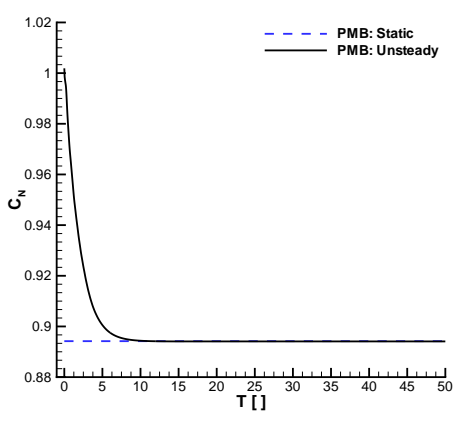

(b) Normal force coefficient, $\alpha=20.0^{\circ}$

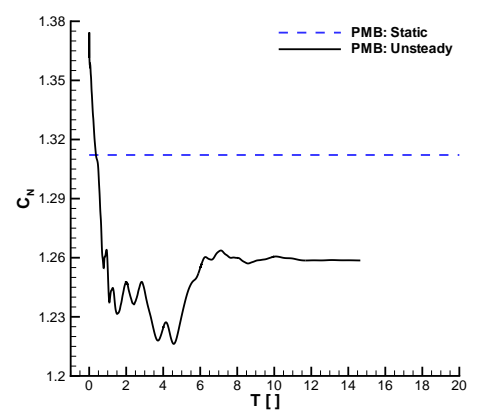

(e) Normal force coefficient, $\alpha=35.0^{\circ}$

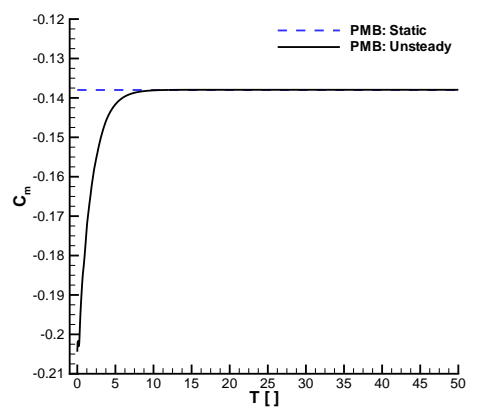

(h) Pitch moment coefficient, $\alpha=20.0^{\circ}$

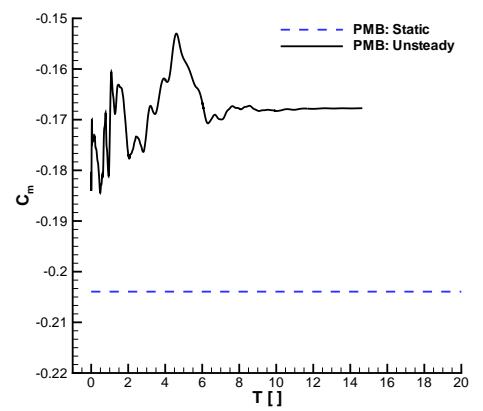

(k) Pitch moment coefficient, $\alpha=35.0^{\circ}$

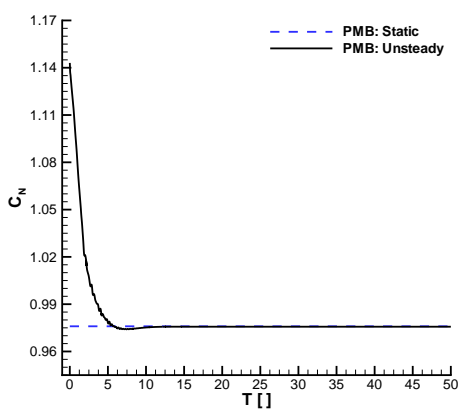

(c) Normal force coefficient, $\alpha=25.0^{\circ}$

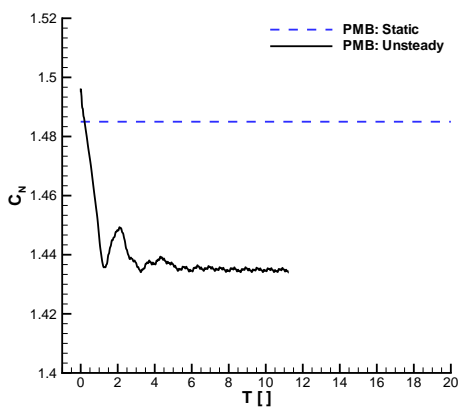

(f) Normal force coefficient, $\alpha=40.0^{\circ}$

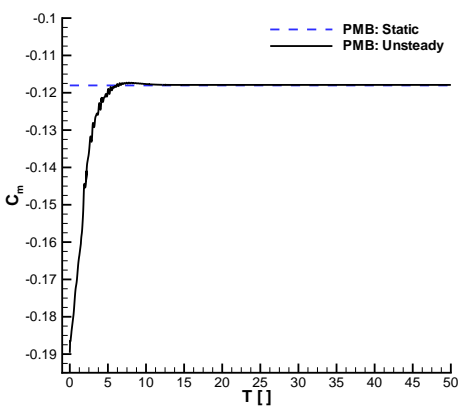

(i) Pitch moment coefficient, $\alpha=25.0^{\circ}$

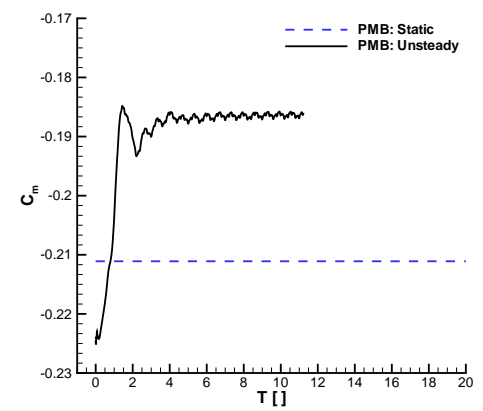

(l) Pitch moment coefficient, $\alpha=40.0^{\circ}$

Figure 21. Normal force and pitching moment coefficient for the TCR fixed geometry unsteady calculations for several angles of incidence 